\title{
GENERALIZED PRODUCT-FORM STATIONARY DISTRIBUTIONS FOR MARKOV CHAINS IN RANDOM ENVIRONMENTS WITH QUEUEING APPLICATIONS
}

\author{
ANTONIS ECONOMOU, ${ }^{*}$ University of Athens
}

\begin{abstract}
Consider a continuous-time Markov chain evolving in a random environment. We study certain forms of interaction between the process of interest and the environmental process, under which the stationary joint distribution is tractable. Moreover, we obtain necessary and sufficient conditions for a product-form stationary distribution. A number of examples that illustrate the applicability of our results in queueing and population growth models are also included.
\end{abstract}

Keywords: Continuous-time Markov chain; random environment; queueing; productform stationary distribution; partial balance; potential; resolvent; ESTA property

2000 Mathematics Subject Classification: Primary 60J22; 65C40

Secondary 60K37; 60J27

\section{Introduction}

In many areas of application of stochastic modelling we find processes that evolve under the influence of a random environment. Several authors have investigated the properties of such models, either in a general setting (e.g. Bourgin and Cogburn (1981), Cogburn and Torrez (1981), and Cogburn (1991)), or in specific areas such as reliability (e.g. Anisimov and Sztrik (1989), Gupta and Gupta (1990), and Lefèvre and Milhaud (1990)), mathematical biology (e.g. Hambly (1992)), mathematical programming (e.g. Helm and Waldmann (1984) and Posner and Zuckerman (1990)), and queueing theory (see below). The majority of such models in the applied probability literature are described by Markov processes evolving in a random environment. Fakinos (1991) and Yamazaki and Miyazawa (1995) study some models within the framework of generalized semi-Markov processes in a random environment.

In the present work, we limit ourselves to the usual framework of continuous-time Markov chains. The general model is a stochastic process $\{(E(t), X(t)): t \geq 0\}$, where $E(t)$ and $X(t)$ represent the random environment and the process of interest, respectively. The main assumption is that the evolution of $X(t)$ does not influence the evolution of $E(t)$, while the evolution of $E(t)$ does influence the evolution of $X(t)$. The environmental process $E(t)$ can influence the evolution of $X(t)$ in the following two ways:

1. The rates at which certain transitions in $X(t)$ occur depend on the environmental state. Thus, a change in the environment might not immediately trigger a transition of $X(t)$, but changes its dynamics (indirect interaction).

2. An environmental change does trigger an immediate transition of $X(t)$ (direct interaction).

Received 6 January 2003; revision received 9 November 2004.

* Postal address: Department of Mathematics, University of Athens, Panepistemiopolis, Athens 15784, Greece.

Email address: aeconom@math.uoa.gr 
Of course, $E(t)$ can influence $X(t)$ in both ways. Most of the reported work concerns models with indirect interaction. In this work we deal with the general case. Our main focus is to shed light on the stationary distributions of such models. Several authors have considered the same problem, using a variety of approaches - matrix-analytic methods seem to be the most widely used. Neuts (1981) devotes his Chapter 6 to applications of such methods in studying queues in random environments and summarizes many of the early results in the subject. More recently, Gaver et al. (1984), O'Cinneide and Purdue (1986), Chang and Nelson (1993), and Núñez-Queija (1997) used such methods to analyse the equilibrium behaviour of diverse queueing systems. More specifically, the $\mathrm{M} / \mathrm{M} / 1, \mathrm{M} / \mathrm{M}^{Y} / 1$, and $\mathrm{M}^{X} / \mathrm{M} / 1$ queues in a random environment lead respectively to quasi-birth-death-, GI/M/1-, and M/G/1-type processes that can be studied effectively using matrix-analytic methods. An alternative approach to analysing similar models is the so-called eigenvalue, or spectral decomposition, method (see, e.g. Mitrani and Chakka (1995)).

Both of the above methods give very satisfactory, exact results whenever they are applicable. However, they apply only in cases where the dynamics of $X(t)$ under the various environmental states are similar, i.e. they are of the same type and differ only in some parameters. Moreover, they demand strong computational power since they require a great number of matrix computations. For these reasons, several authors have tried to identify some categories of model in which the stationary distributions assume a simple product form. More specifically, Sztrik (1987), Falin (1996), Zhu (1991), (1994), and Tsitsiashvili et al. (2002) have identified conditions that ensure product-form stationary distributions for several concrete classes of queueing systems in random environments. In the present paper, we study the same problem within a general framework and we state necessary and sufficient conditions for product-form distributions. Moreover, whenever these conditions fail, we develop an alternative approach for computing the stationary distributions for several classes of model, using the notion of the potential (or resolvent) of a continuous-time Markov chain. This approach is based on an inversion formula that expresses the relationship between the stationary distribution of the original Markov chain and the stationary distribution of its embedded chains (Palm distributions) at certain environmental change epochs. Thus, we also study the events-see-time-averages (ESTA) property within this class of model.

The outline of the paper is as follows. After describing the general model and motivating the problems we consider, in Section 2, we prove the necessary and sufficient conditions for a product-form stationary distribution in Section 3. In Section 4, we treat some other tractable cases where the stationary distributions of the models can be obtained in closed form. In Sections 5 and 6, we present several applications of the product-form results and the inversion formula, respectively.

\section{The general model}

In this section, we define a general structure for a continuous-time Markov chain evolving in a random environment. The model is an ergodic (i.e. irreducible and positive-recurrent) Markov chain $\{(E(t), X(t)): t \geq 0\}$ with state space $\mathcal{E} \times \mathcal{X}$, where $E(t)$ and $X(t)$ represent the random environment and the process of interest, respectively. We assume that $E(t)$ jumps from state to state according to an ergodic continuous-time Markov chain transition rate matrix $\boldsymbol{Q}_{E}=\left(q_{E}\left(e, e^{\prime}\right): e, e^{\prime} \in \mathcal{E}\right)$. Upon an environmental change from $e$ to $e^{\prime}$, the process $X(t)$ at state $x$ jumps immediately to some state $x^{\prime} \in \mathcal{X}$ with probability $\alpha\left(x^{\prime} \mid e, x, e^{\prime}\right)$. The discrete probability density $\left(\alpha\left(x^{\prime} \mid e, x, e^{\prime}\right): x^{\prime} \in \mathcal{X}\right)$ will be referred to as the triggering probability function for the state $x$ and the environmental transition $e \rightarrow e^{\prime}$. 
In the meantime, between two successive environmental transitions, the process $X(t)$ is governed by the transition rate matrix $Q_{X \mid E}(e)=\left(q_{X \mid E}\left(x, x^{\prime} \mid e\right): x, x^{\prime} \in \mathcal{X}\right)$ of an irreducible Markov chain on $\mathcal{X}$, where $e$ is the current environmental state. More concretely, the transition rates $q\left((e, x),\left(e^{\prime}, x^{\prime}\right)\right)$ of $(E(t), X(t))$ are given by

$$
q\left((e, x),\left(e^{\prime}, x^{\prime}\right)\right)= \begin{cases}q_{X \mid E}\left(x, x^{\prime} \mid e\right) & \text { if } e^{\prime}=e, x^{\prime} \neq x, \\ q_{E}\left(e, e^{\prime}\right) \alpha\left(x^{\prime} \mid e, x, e^{\prime}\right) & \text { if } e^{\prime} \neq e .\end{cases}
$$

Let $\pi=(\pi(e, x): e \in \mathcal{E}, x \in \mathcal{X})$ be the joint stationary distribution of $\{(E(t), X(t))\}$ and $\pi_{E}=\left(\pi_{E}(e): e \in \mathcal{E}\right), \pi_{X}=\left(\pi_{X}(x): x \in \mathcal{X}\right)$ its marginal distributions. Furthermore, let $\boldsymbol{P}_{X \mid E}^{(t)}(e)=\left(p_{X \mid E}^{(t)}\left(x, x^{\prime} \mid e\right): x, x^{\prime} \in \mathcal{X}\right)$ be the transition probability matrix at time $t$ for the Markov chain with rate matrix $\boldsymbol{Q}_{X \mid E}(e)$, and let $\pi_{X \mid E}(e)=\left(\pi_{X \mid E}(x \mid e): x \in \mathcal{X}\right)$ be its stationary distribution (in the ergodic case in which it exists and is unique). We are interested in determining $\boldsymbol{\pi}, \boldsymbol{\pi}_{E}$, and $\boldsymbol{\pi}_{X}$ and in examining their dependences on the transition rate matrices $\boldsymbol{Q}_{E}$ and $\boldsymbol{Q}_{X \mid E}(e), e \in \mathcal{E}$. We are also interested in studying the Palm (or embedded) distributions of $X(t)$ just after (or before) certain environmental transitions. More specifically, for every $e \in \mathcal{E}$, let $t_{0}(e)<t_{1}(e)<t_{2}(e)<\cdots$ be the successive times that $E(t)$ 'arrives' at state $e$, and $\tau_{0}(e)<\tau_{1}(e)<\tau_{2}(e)<\cdots$ be the successive times that $E(t)$ 'departs' from state $e$. Define the embedded processes $\left\{X_{\mathrm{a}(e)}(n): n \geq 0\right\}$ and $\left\{X_{\mathrm{d}(e)}(n): n \geq 0\right\}$ of $\{X(t)\}$ by $X_{\mathrm{a}(e)}(n)=X\left(t_{n}(e)\right)$ and $X_{\mathrm{d}(e)}(n)=X\left(\tau_{n}^{-}(e)\right)$, i.e. $X_{\mathrm{a}(e)}(n)$ is the state of $\{X(t)\}$ just after the $n$th environmental arrival to $e$, while $X_{\mathrm{d}(e)}(n)$ is the state of $\{X(t)\}$ just before the $n$th environmental departure from $e$. Let $\pi_{\mathrm{a}(e)}=\left(\pi_{\mathrm{a}(e)}(x): x \in \mathcal{X}\right)$ and $\pi_{\mathrm{d}(e)}=\left(\pi_{\mathrm{d}(e)}(x): x \in \mathcal{X}\right)$ be the stationary distributions of $\left\{X_{\mathrm{a}(e)}(n)\right\}$ and $\left\{X_{\mathrm{d}(e)}(n)\right\}$, respectively. Since the jump rate from state $(e, x)$ to $\left(e^{\prime}, x^{\prime}\right)$ is $\pi(e, x) q\left((e, x),\left(e^{\prime}, x^{\prime}\right)\right)$, we have (see Section 2.2 of Chao et al. (1999) for details) that these Palm distributions are given by

$$
\begin{aligned}
& \pi_{\mathrm{a}(e)}(x)=\frac{\sum_{x^{\prime}} \sum_{e^{\prime} \neq e} \pi\left(e^{\prime}, x^{\prime}\right) q_{E}\left(e^{\prime}, e\right) \alpha\left(x \mid e^{\prime}, x^{\prime}, e\right)}{\sum_{y} \sum_{x^{\prime}} \sum_{e^{\prime} \neq e} \pi\left(e^{\prime}, x^{\prime}\right) q_{E}\left(e^{\prime}, e\right) \alpha\left(y \mid e^{\prime}, x^{\prime}, e\right)}, \\
& \pi_{\mathrm{d}(e)}(x)=\frac{\sum_{x^{\prime}} \sum_{e^{\prime} \neq e} \pi(e, x) q_{E}\left(e, e^{\prime}\right) \alpha\left(x^{\prime} \mid e, x, e^{\prime}\right)}{\sum_{y} \sum_{x^{\prime}} \sum_{e^{\prime} \neq e} \pi(e, y) q_{E}\left(e, e^{\prime}\right) \alpha\left(x^{\prime} \mid e, y, e^{\prime}\right)} .
\end{aligned}
$$

It is known that the Palm distributions of a process that correspond to different sets of transitions neither coincide with one another nor with the stationary distribution of the process, in general. In such cases, it is important to study the relationships of these distributions and also to find conditions under which they do coincide (ESTA property). There are many such results in the literature, concerning a variety of processes; see, for example, Melamed and Yao (1995), Stidham and El-Taha (1995), and El-Taha and Stidham (1999). We will study the ESTA problem within the present framework in Section 3.

The stationary distribution $\pi=(\pi(e, x))$ of $\{(E(t), X(t))\}$ satisfies the (full) balance equations

$$
\begin{aligned}
& \pi(e, x)\left(\sum_{e^{\prime} \neq e} q_{E}\left(e, e^{\prime}\right)+\sum_{x^{\prime} \neq x} q_{X \mid E}\left(x, x^{\prime} \mid e\right)\right) \\
&= \sum_{e^{\prime} \neq e} \sum_{x^{\prime}} \pi\left(e^{\prime}, x^{\prime}\right) q_{E}\left(e^{\prime}, e\right) \alpha\left(x \mid e^{\prime}, x^{\prime}, e\right)+\sum_{x^{\prime} \neq x} \pi\left(e, x^{\prime}\right) q_{X \mid E}\left(x^{\prime}, x \mid e\right), \\
& e \in \mathcal{E}, x \in X .
\end{aligned}
$$


By summing these equations over $x$ for every environmental state $e$, we obtain

$$
\pi_{E}(e) \sum_{e^{\prime} \neq e} q_{E}\left(e, e^{\prime}\right)=\sum_{e^{\prime} \neq e} \pi_{E}\left(e^{\prime}\right) q_{E}\left(e^{\prime}, e\right), \quad e \in \mathcal{E} .
$$

Therefore, the marginal distribution $\pi_{E}=\left(\pi_{E}(e)\right)$ satisfies the balance equations of the Markov chain with transition rate matrix $\boldsymbol{Q}_{E}=\left(q_{E}\left(e, e^{\prime}\right)\right)$. By the ergodicity of this Markov chain, which implies the uniqueness of its stationary distribution, we obtain the following lemma.

Lemma 1. The marginal distribution $\pi_{E}=\left(\pi_{E}(e)\right)$ is the stationary distribution of the Markov chain with transition rate matrix $\boldsymbol{Q}_{E}=\left(q_{E}\left(e, e^{\prime}\right)\right)$.

Using Lemma 1 and the fact that $\sum_{x^{\prime} \in \mathcal{X}} \alpha\left(x^{\prime} \mid e, x, e^{\prime}\right)=1$ for every $x \in \mathcal{X}$ and $e, e^{\prime} \in \mathcal{E}$, (2) and (3) simplify respectively to

$$
\begin{aligned}
& \pi_{\mathrm{a}(e)}(x)=\frac{\sum_{x^{\prime}} \sum_{e^{\prime} \neq e} \pi\left(e^{\prime}, x^{\prime}\right) q_{E}\left(e^{\prime}, e\right) \alpha\left(x \mid e^{\prime}, x^{\prime}, e\right)}{\pi_{E}(e) q_{E}(e)}, \\
& \pi_{\mathrm{d}(e)}(x)=\frac{\pi(e, x)}{\pi_{E}(e)},
\end{aligned}
$$

where $q_{E}(e)=\sum_{e^{\prime} \neq e} q_{E}\left(e, e^{\prime}\right)$.

We will now present some special types of triggering probability function

$$
\boldsymbol{\alpha}_{e, x, e^{\prime}}=\left(\alpha\left(x^{\prime} \mid e, x, e^{\prime}\right): x^{\prime} \in \mathcal{X}\right)
$$

that appear in the applications and that are used in the following sections.

Type 1. $\alpha\left(x^{\prime} \mid e, x, e^{\prime}\right)=\mathbf{1}_{\{x\}}\left(x^{\prime}\right)= \begin{cases}1 & \text { if } x^{\prime}=x, \\ 0 & \text { if } x^{\prime} \neq x\end{cases}$

The indirect interaction that we described loosely in the Introduction is of this type: the environmental process influences the dynamics of $X(t)$ but does not trigger immediate events. In this case, (5) assumes the form

$$
\pi_{\mathrm{a}(e)}(x)=\frac{\sum_{e^{\prime} \neq e} \pi\left(e^{\prime}, x\right) q_{E}\left(e^{\prime}, e\right)}{\pi_{E}(e) q_{E}(e)} .
$$

Type 2. $\alpha\left(x^{\prime} \mid e, x, e^{\prime}\right)=\gamma\left(x^{\prime} \mid e, e^{\prime}\right)$.

For this type of triggering probability function, after an environmental change from $e$ to $e^{\prime}$, $X(t)$ forgets its state and starts anew depending on $e$ and $e^{\prime}$. In this case, (5) assumes the form

$$
\pi_{\mathrm{a}(e)}(x)=\frac{\sum_{e^{\prime} \neq e} \pi_{E}\left(e^{\prime}\right) q_{E}\left(e^{\prime}, e\right) \gamma\left(x \mid e^{\prime}, e\right)}{\pi_{E}(e) q_{E}(e)} .
$$

Type 3. $\alpha\left(x^{\prime} \mid e, x, e^{\prime}\right)=\beta\left(x^{\prime} \mid e^{\prime}\right)$.

This is a special case of type 2 , for which $X(t)$ starts anew depending only on the new environmental state $e^{\prime}$. In this case, (5) assumes the form

$$
\pi_{\mathrm{a}(e)}(x)=\beta(x \mid e) .
$$


Type 4. $\alpha\left(x^{\prime} \mid e, x, e^{\prime}\right)=\pi_{X \mid E}\left(x^{\prime} \mid e^{\prime}\right)$.

This is a special case of type 3 , for which $X(t)$ starts in equilibrium after every environmental change. To picture this case, imagine that there exists an experiment that is performed concurrently in $|\mathscr{E}|$ positions with different environmental conditions, with an observer that moves randomly from position to position and records a certain variable of the experiment. Then, $E(t)$ is the position of the observer and $X(t)$ is the recorded value of the variable at time $t$. The evolution of the experiment at each of the $|\mathcal{E}|$ positions is assumed to be in equilibrium and independent of the evolution at every other position. The observer does not influence the experiments. In this case, (5) assumes the form

$$
\pi_{\mathrm{a}(e)}(x)=\pi_{X \mid E}(x \mid e) .
$$

\section{Product-form distributions}

Equations (4) can be decomposed into the following partial balance equations, which are not satisfied by $\pi$, in general:

$$
\begin{aligned}
& \pi(e, x) \sum_{e^{\prime} \neq e} q_{E}\left(e, e^{\prime}\right)=\sum_{e^{\prime} \neq e} \sum_{x^{\prime}} \pi\left(e^{\prime}, x^{\prime}\right) q_{E}\left(e^{\prime}, e\right) \alpha\left(x \mid e^{\prime}, x^{\prime}, e\right), \quad e \in \mathcal{E}, x \in \mathcal{X}, \\
& \pi(e, x) \sum_{x^{\prime} \neq x} q_{X \mid E}\left(x, x^{\prime} \mid e\right)=\sum_{x^{\prime} \neq x} \pi\left(e, x^{\prime}\right) q_{X \mid E}\left(x^{\prime}, x \mid e\right), \quad e \in \mathcal{E}, x \in \mathcal{X} .
\end{aligned}
$$

If the partial balance equations (7) are satisfied then, for every state $(e, x)$, the rate out of it due to an environmental change equals the rate into it due to an environmental change. Similarly, the partial balance equations (8), whenever satisfied, express a balance between the rate out of a specific state $(e, x)$, due to a nonenvironmental change, and the rate into the same state due to a nonenvironmental change.

The phenomenon of partial balance, and its implications, have been extensively studied in the literature. Kelly (1979) summarizes the early results about the subject, while van Dijk (1993), Chao et al. (1999), and Serfozo (1999) contain recent results and many queueing applications. It has been generally noted that the presence of partial balance facilitates the study of a given model. First, it implies the equality of the Palm distributions at certain event epochs (see, for example, Theorem 9.5 of Kelly (1979) and Theorem 1 of Fakinos and Economou (1998)). Second, under certain additional conditions, it implies that the stationary distribution assumes a certain product form. In the following theorem we investigate the phenomenon of partial balance within the framework of our model.

Theorem 1. For the general model, with transition rates given by (1), the following statements are equivalent.

(i) The Palm distributions $\pi_{\mathrm{a}(e)}$ and $\pi_{\mathrm{d}(e)}$ coincide for every $e \in \mathcal{E}$.

(ii) The stationary distribution $\pi$ satisfies the partial balance equations (7).

(iii) The stationary distribution $\pi$ satisfies the partial balance equations (8).

If, moreover, the transition matrices $\boldsymbol{Q}_{X \mid E}($ e) are ergodic for all $e \in \mathcal{E}$, then (i)-(iii) are also equivalent to two further statements:

(iv) The stationary distribution $\pi$ is given by the product-form formula

$$
\pi(e, x)=\pi_{E}(e) \pi_{X \mid E}(x \mid e), \quad e \in \mathcal{E}, x \in \mathcal{X} .
$$


(v) The Palm distribution $\pi_{\mathrm{d}(e)}$ coincides with the stationary distribution $\pi_{X \mid E}(e)$ for every $e \in \mathcal{E}$.

If the conditions $(i)-(v)$ are satisfied and the additional conditions

$$
\pi_{X \mid E}(x \mid e)=\sum_{x^{\prime} \in \mathcal{X}} \pi_{X \mid E}\left(x^{\prime} \mid e^{\prime}\right) \alpha\left(x \mid e^{\prime}, x^{\prime}, e\right), \quad e, e^{\prime} \in \mathcal{E}, x \in \mathcal{X},
$$

hold, then the reversed process of $(E(t), X(t))$, namely $\left(E^{\mathrm{R}}(t), X^{\mathrm{R}}(t)\right)$, is of the same type, i.e. its transition rates $\left(q^{\mathrm{R}}\left((e, x),\left(e^{\prime}, x^{\prime}\right)\right)\right)$ are of the form (1) and, more concretely,

$$
q^{\mathrm{R}}\left((e, x),\left(e^{\prime}, x^{\prime}\right)\right)= \begin{cases}q_{X \mid E}^{\mathrm{R}}\left(x, x^{\prime} \mid e\right) & \text { if } e^{\prime}=e, x^{\prime} \neq x, \\ q_{E}^{\mathrm{R}}\left(e, e^{\prime}\right) \tilde{\alpha}\left(x^{\prime} \mid e, x, e^{\prime}\right) & \text { if } e^{\prime} \neq e,\end{cases}
$$

where $\left(q_{X \mid E}^{\mathrm{R}}\left(x, x^{\prime} \mid e\right): x, x^{\prime} \in \mathcal{X}\right)$ are the reversed rates of $\left(q_{X \mid E}\left(x, x^{\prime} \mid e\right): x, x^{\prime} \in \mathcal{X}\right)$ for every $e \in \mathcal{E},\left(q_{E}^{\mathrm{R}}\left(e, e^{\prime}\right): e, e^{\prime} \in \mathcal{E}\right)$ are the reversed rates of $\left(q_{E}\left(e, e^{\prime}\right): e, e^{\prime} \in \mathcal{E}\right)$, and

$$
\tilde{\alpha}\left(x^{\prime} \mid e, x, e^{\prime}\right)=\frac{\pi_{X \mid E}\left(x^{\prime} \mid e^{\prime}\right) \alpha\left(x \mid e^{\prime}, x^{\prime}, e\right)}{\pi_{X \mid E}(x \mid e)}, \quad e, e^{\prime} \in \mathcal{E}, x, x^{\prime} \in \mathcal{X} .
$$

Proof. (i) $\Leftrightarrow$ (ii). The probabilities $\pi_{\mathrm{a}(e)}(x)$ and $\pi_{\mathrm{d}(e)}(x)$ given by (5) and (6) are respectively equal to the right- and the left-hand sides of the partial balance equations (7) divided by $\pi_{E}(e) q_{E}(e)$.

(ii) $\Leftrightarrow$ (iii). This is immediate, in light of the full balance equations (4).

(iii) $\Rightarrow$ (iv). Consider a fixed $e \in \mathcal{E}$. Then, by (8), the vector $(\pi(e, x): x \in \mathcal{X})$ satisfies the balance equations of the Markov chain with transition rate matrix $\boldsymbol{Q}_{X \mid E}(e)$. Therefore, $(\pi(e, x): x \in \mathcal{X})$ is a scalar multiple of the stationary distribution $\pi_{X \mid E}(e)=\left(\pi_{X \mid E}(x \mid e)\right.$ : $x \in \mathcal{X})$ of $\boldsymbol{Q}_{X \mid E}$, that is, $\pi(e, x)=c(e) \pi_{X \mid E}(x \mid e)$. By summing over $x$, we obtain that $\pi_{E}(e)=c(e)$; hence, $\pi(e, x)$ assumes the form (9).

(iv) $\Rightarrow$ (iii). Substitute $\pi(e, x)$, given by (9), into (8) and divide by $\pi_{E}(e)$. The resulting equations are the balance equations for $\boldsymbol{Q}_{X \mid E}(e)$.

(iv) $\Leftrightarrow$ (v). This is immediate, in light of (6).

When conditions (i) $-(\mathrm{v})$ hold, the reversed rates $q^{\mathrm{R}}\left((e, x),\left(e^{\prime}, x^{\prime}\right)\right)$ can be directly computed, and we obtain (11). Condition (10) ensures that $\left(\tilde{\alpha}\left(x^{\prime} \mid e, x, e^{\prime}\right): x^{\prime} \in \mathcal{X}\right)$ is a probability function.

Remark 1. Theorem 1 completely characterizes the partial balance for the general model. Moreover, in the important special case where all matrices $\boldsymbol{Q}_{X \mid E}(e)$ are ergodic, the theorem suggests the following approach to checking partial balance in a given model. First, compute the stationary distributions $\pi_{E}$ and $\pi_{X \mid E}(e), e \in \mathcal{E}$. Second, check whether the distribution $\left(\pi_{E}(e) \pi_{X \mid E}(x \mid e): e \in \mathcal{E}, x \in \mathcal{X}\right)$ satisfies the full balance equations (4) or, equivalently, the partial balance equations (7) (noting that it always satisfies the partial balance equations (8)). If it does then conditions (i)-(v) of Theorem 1 are satisfied and we have that the stationary distribution $\pi$ of the model is given by (9), while $\pi_{\mathrm{a}(e)}=\pi_{\mathrm{d}(e)}=\pi_{X \mid E}(e)$ for every $e \in \mathcal{E}$.

By applying Theorem 1 in models with type-1 or type-3 triggering probability functions, we obtain the following corollaries. 
Corollary 1. Consider the model with transition rates given by (1) and type-1 triggering probability function $\alpha\left(x^{\prime} \mid e, x, e^{\prime}\right)=\mathbf{1}_{\{x\}}\left(x^{\prime}\right)$, with all matrices $Q_{X \mid E}(e)$ being ergodic. The following statements are equivalent.

(i) The stationary distribution $\pi$ is given by the product-form formula (9).

(ii) In stationarity, $E(t)$ and $X(t)$ are independent, that is, $\pi$ has the form

$$
\pi(e, x)=\pi_{E}(e) \pi_{X}(x), \quad e \in \mathcal{E}, x \in \mathcal{X} .
$$

(iii) All the stationary distributions $\boldsymbol{\pi}_{X \mid E}(e)$ coincide.

Under conditions ( $i)-\left(\right.$ iii), the reversed process $\left(E^{\mathrm{R}}(t), X^{\mathrm{R}}(t)\right)$ has transition rates given by

$$
q^{\mathrm{R}}\left((e, x),\left(e^{\prime}, x^{\prime}\right)\right)= \begin{cases}q_{X \mid E}^{\mathrm{R}}\left(x, x^{\prime} \mid e\right) & \text { if } e^{\prime}=e, x^{\prime} \neq x, \\ q_{E}^{\mathrm{R}}\left(e, e^{\prime}\right) & \text { if } e^{\prime} \neq e, x^{\prime}=x .\end{cases}
$$

Proof. (i) $\Rightarrow$ (ii). The equivalent conditions (i)-(v) of Theorem 1 are satisfied. By Theorem 1(ii), we have that the stationary distribution $\pi$ satisfies (7), which, in this case, assumes the form

$$
\pi(e, x) \sum_{e^{\prime} \neq e} q_{E}\left(e, e^{\prime}\right)=\sum_{e^{\prime} \neq e} \pi\left(e^{\prime}, x\right) q_{E}\left(e^{\prime}, e\right), \quad e \in \mathcal{E}, x \in \mathcal{X} .
$$

For a fixed $x \in \mathcal{X}$, the above equations show that $(\pi(e, x): e \in \mathcal{E})$ satisfies the balance equations of the Markov chain with transition rate matrix $Q_{E}$. Therefore, $(\pi(e, x): e \in \mathcal{E})$ is a scalar multiple of the stationary distribution $\pi_{E}$, that is, $\pi(e, x)=c(x) \pi_{E}(e)$. By summing over $e$, we obtain $c(x)=\pi_{X}(x)$ and $\pi(e, x)$ assumes the form (12).

(ii) $\Rightarrow$ (iii). The distribution $(\pi(e, x))$ given by (12) satisfies (4) and (7). Therefore, it also satisfies (8). We divide by $\pi_{E}(e)$ to obtain

$$
\pi_{X}(x) \sum_{x^{\prime} \neq x} q_{X \mid E}\left(x, x^{\prime} \mid e\right)=\sum_{x^{\prime} \neq x} \pi_{X}\left(x^{\prime}\right) q_{X \mid E}\left(x^{\prime}, x \mid e\right), \quad e \in \mathcal{E}, x \in \mathcal{X} .
$$

For a fixed $e \in \mathcal{E}$, these equations show that $\left(\pi_{X}(x): x \in \mathcal{X}\right)$ is the stationary distribution of $\boldsymbol{Q}_{X \mid E}$. Therefore $\boldsymbol{\pi}_{X \mid E}(e)=\boldsymbol{\pi}_{X}$ for every $e \in \mathcal{E}$, i.e. all the stationary distributions $\boldsymbol{\pi}_{X \mid E}(e)$, $e \in \mathcal{E}$, coincide.

(iii) $\Rightarrow$ (i). Let $\pi_{X \mid E}(x \mid e)=c(x), e \in \mathcal{E}, x \in \mathcal{X}$. Then, the distribution $\left(\pi_{E}(e) c(x): e \in \mathcal{E}\right.$, $x \in \mathcal{X}$ ) is easily seen to satisfy the balance equations (4). Therefore, condition (iv) of Theorem 1 is valid and we obtain the result.

For this type of triggering probability function, condition (10) is obviously valid, and (11) is reduced to (13).

Corollary 2. Consider the model with transition rates given by (1) and type-3 triggering probability function $\alpha\left(x^{\prime} \mid e, x, e^{\prime}\right)=\beta\left(x^{\prime} \mid e^{\prime}\right)$, with all matrices $\boldsymbol{Q}_{X \mid E}(e)$ being ergodic. The following statements are equivalent.

(i) The stationary distribution $\pi$ is given by the product-form formula (9).

(ii) The distributions $(\beta(x \mid e): x \in \mathcal{X})$ and $\left(\pi_{X \mid E}(x \mid e): x \in \mathcal{X}\right)$ coincide for everye $\in \mathcal{E}$. 
Under conditions $(i)$ and (ii), the reversed process $\left(E^{\mathrm{R}}(t), X^{\mathrm{R}}(t)\right)$ has transition rates $q^{\mathrm{R}}\left((e, x),\left(e^{\prime}, x^{\prime}\right)\right)$ given by

$$
q^{\mathrm{R}}\left((e, x),\left(e^{\prime}, x^{\prime}\right)\right)= \begin{cases}q_{X \mid E}^{\mathrm{R}}\left(x, x^{\prime} \mid e\right) & \text { if } e^{\prime}=e, x^{\prime} \neq x, \\ q_{E}^{\mathrm{R}}\left(e, e^{\prime}\right) \beta\left(x^{\prime} \mid e^{\prime}\right) & \text { if } e^{\prime} \neq e .\end{cases}
$$

Proof. (i) $\Rightarrow$ (ii). The equivalent conditions (i)-(v) of Theorem 1 are satisfied. Therefore, the stationary distribution $\pi$ satisfies (7), which, in this case, reduces to

$$
\pi(e, x)=\pi_{E}(e) \beta(x \mid e), \quad e \in \mathcal{E}, x \in \mathcal{X} .
$$

However, $\pi(e, x)$ is also given by (9), so we obtain

$$
\pi_{X \mid E}(x \mid e)=\beta(x \mid e), \quad e \in \mathcal{E}, x \in \mathcal{X} .
$$

(ii) $\Rightarrow$ (i). The distribution $\pi$ given by (9) always satisfies the partial balance equations (8). In the case where $\pi_{X \mid E}(x \mid e)=\beta(x \mid e)$, it is readily seen to satisfy the partial balance equations (7) also and, hence, to be the stationary distribution of the model.

For this type of triggering probability function, condition (10) is reduced to $\pi_{X \mid E}(x \mid e)=$ $\beta(x \mid e)$, which is valid by statement (ii) of Corollary 2 . Therefore, (11) applies with

$$
\tilde{\alpha}\left(x^{\prime} \mid e, x, e^{\prime}\right)=\beta\left(x^{\prime} \mid e^{\prime}\right),
$$

and we obtain (14).

\section{The inversion formula}

Theorem 1 and Corollaries 1 and 2 exactly describe the relationships between the stationary distribution $\pi$ and the Palm distributions $\pi_{\mathrm{a}(e)}$ and $\pi_{\mathrm{d}(e)}, e \in \mathcal{E}$, when partial balance holds. Unfortunately, the models that fall into the framework of Theorem 1 are very limited. Therefore, in the general case, we rely solely on (5) and (6). It is clear that if we know $\pi$ or $\left(\pi_{\mathrm{d}(e)}: e \in \mathcal{E}\right)$, we can easily obtain $\left(\pi_{\mathrm{a}(e)}: e \in \mathcal{E}\right)$. It is not obvious how to invert (5) and obtain $\pi$ and $\left(\pi_{\mathrm{d}(e)}: e \in \mathcal{E}\right)$ when $\left(\pi_{\mathrm{a}(e)}: e \in \mathcal{E}\right)$ is known, but in Theorem 2 , below, we provide just such an inversion formula. Apart from the theoretical interest of the inversion formula, having one will enable us easily to compute the stationary distribution $\pi$ of any model with triggering probability function of type 2 or 3 . Moreover, it is the basis of a powerful computational procedure for computing the stationary distributions for a broad class of models.

Theorem 2. (Inversion formula.) Given the Palm distributions $\boldsymbol{\pi}_{\mathrm{a}(e)}$, the stationary distribution $\pi$ can be computed as follows:

$$
\pi(e, x)=\pi_{E}(e) q_{E}(e) \int_{0}^{\infty} \mathrm{e}^{-q_{E}(e) t} \pi_{\mathrm{a}(e)}^{(t)}(x) \mathrm{d} t, \quad e \in \mathcal{E}, x \in \mathcal{X},
$$

where $\pi_{\mathrm{a}(e)}^{(t)}=\left(\pi_{\mathrm{a}(e)}^{(t)}(x): x \in \mathcal{X}\right)$ is the transient probability function at time $t$ of a Markov chain with initial distribution $\boldsymbol{\pi}_{\mathrm{a}(e)}$ and transition rate matrix $\boldsymbol{Q}_{X \mid E}(e)$.

Proof. By using (5) and $\pi_{\mathrm{a}(e)}^{(t)}(x)=\sum_{y \in X} \pi_{\mathrm{a}(e)}(y) p_{X \mid E}^{(t)}(y, x \mid e)$, we obtain

$$
\begin{aligned}
& \pi_{E}(e) q_{E}(e) \int_{0}^{\infty} \mathrm{e}^{-q_{E}(e) t} \pi_{\mathrm{a}(e)}^{(t)}(x) \mathrm{d} t \\
& =\sum_{y \in \mathcal{X}} \sum_{x^{\prime} \in \mathcal{X}} \sum_{e^{\prime} \neq e} \pi\left(e^{\prime}, x^{\prime}\right) q_{E}\left(e^{\prime}, e\right) \alpha\left(y \mid e^{\prime}, x^{\prime}, e\right) \int_{0}^{\infty} \mathrm{e}^{-q_{E}(e) t} p_{X \mid E}^{(t)}(y, x \mid e) \mathrm{d} t .
\end{aligned}
$$


By the full balance equations (4), we have that

$$
\begin{aligned}
\sum_{e^{\prime} \neq e} \sum_{x^{\prime}} \pi\left(e^{\prime}, x^{\prime}\right) q_{E}\left(e^{\prime}, e\right) \alpha\left(y \mid e^{\prime}, x^{\prime}, e\right) & \\
= & \pi(e, y) q_{E}(e)+\pi(e, y) \sum_{x^{\prime} \neq y} q_{X \mid E}\left(y, x^{\prime} \mid e\right) \\
& -\sum_{x^{\prime} \neq y} \pi\left(e, x^{\prime}\right) q_{X \mid E}\left(x^{\prime}, y \mid e\right), \quad e \in \mathcal{E}, x \in \mathcal{X},
\end{aligned}
$$

and, therefore, (16) becomes

$$
\begin{aligned}
\pi_{E}(e) q_{E}(e) \int_{0}^{\infty} \mathrm{e}^{-q_{E}(e) t} \pi_{\mathrm{a}(e)}^{(t)}(x) \mathrm{d} t \\
=\sum_{y \in X} \pi(e, y) q_{E}(e) \int_{0}^{\infty} \mathrm{e}^{-q_{E}(e) t} p_{X \mid E}^{(t)}(y, x \mid e) \mathrm{d} t \\
\quad+\sum_{y \in X} \pi(e, y) \sum_{x^{\prime} \neq y} q_{X \mid E}\left(y, x^{\prime} \mid e\right) \int_{0}^{\infty} \mathrm{e}^{-q_{E}(e) t} p_{X \mid E}^{(t)}(y, x \mid e) \mathrm{d} t \\
\quad-\sum_{y \in X} \sum_{x^{\prime} \neq y} \pi\left(e, x^{\prime}\right) q_{X \mid E}\left(x^{\prime}, y \mid e\right) \int_{0}^{\infty} \mathrm{e}^{-q_{E}(e) t} p_{X \mid E}^{(t)}(y, x \mid e) \mathrm{d} t .
\end{aligned}
$$

By using the Chapman-Kolmogorov differential equations for the transition probabilities

$$
p_{X \mid E}^{(t)}(y, x \mid e)
$$

the last term in the right-hand side of (17) can be written equivalently as

$$
\begin{aligned}
\sum_{x^{\prime} \in \mathcal{X}} \pi & \left(e, x^{\prime}\right) \int_{0}^{\infty} \mathrm{e}^{-q_{E}(e) t} \sum_{y \neq x^{\prime}} q_{X \mid E}\left(x^{\prime}, y \mid e\right) p_{X \mid E}^{(t)}(y, x \mid e) \mathrm{d} t \\
= & \sum_{x^{\prime} \in \mathcal{X}} \pi\left(e, x^{\prime}\right) \int_{0}^{\infty} \mathrm{e}^{-q_{E}(e) t} \frac{\mathrm{d}}{\mathrm{d} t} p_{X \mid E}^{(t)}\left(x^{\prime}, x \mid e\right) \mathrm{d} t \\
& +\sum_{x^{\prime} \in \mathcal{X}} \pi\left(e, x^{\prime}\right) \int_{0}^{\infty} \mathrm{e}^{-q_{E}(e) t} \sum_{y \neq x^{\prime}} q_{X \mid E}\left(x^{\prime}, y \mid e\right) p_{X \mid E}^{(t)}\left(x^{\prime}, x \mid e\right) \mathrm{d} t .
\end{aligned}
$$

By substituting (18) into (17), we obtain

$$
\begin{aligned}
\pi_{E}(e) q_{E}(e) \int_{0}^{\infty} \mathrm{e}^{-q_{E}(e) t} \pi_{\mathrm{a}(e)}^{(t)}(x) \mathrm{d} t= & \sum_{y \in \mathcal{X}} \pi(e, y) q_{E}(e) \int_{0}^{\infty} \mathrm{e}^{-q_{E}(e) t} p_{X \mid E}^{(t)}(y, x \mid e) \mathrm{d} t \\
& -\sum_{x^{\prime} \in \mathcal{X}} \pi\left(e, x^{\prime}\right) \int_{0}^{\infty} \mathrm{e}^{-q_{E}(e) t} \frac{\mathrm{d}}{\mathrm{d} t} p_{X \mid E}^{(t)}\left(x^{\prime}, x \mid e\right) \mathrm{d} t .
\end{aligned}
$$

Using integration by parts, the last term is transformed into

$$
\sum_{y \in X} \pi(e, y)\left[\mathrm{e}^{-q_{E}(e) t} p_{X \mid E}^{(t)}(y, x \mid e)\right]_{t=0}^{\infty}+\sum_{y \in \mathcal{X}} \pi(e, y) \int_{0}^{\infty} q_{E}(e) \mathrm{e}^{-q_{E}(e) t} p_{X \mid E}^{(t)}(y, x \mid e) \mathrm{d} t .
$$

By substituting this, in turn, into (19) and simplifying, we arrive at (15). 
Remark 2. The inversion formula (15) can be written equivalently as

$$
\pi(e, x)=\pi_{E}(e) q_{E}(e) \sum_{y \in \mathcal{X}} \pi_{\mathrm{a}(e)}(y) m_{q(e)}(y, x \mid e), \quad e \in \mathcal{E}, x \in \mathcal{X},
$$

where $m_{\alpha}(y, x \mid e)=\int_{0}^{\infty} \mathrm{e}^{-\alpha t} p_{X \mid E}^{(t)}(y, x \mid e) \mathrm{d} t(\alpha>0)$ are the so-called $\alpha$-potentials (or resolvents) of the Markov chain with transition probabilities $\left(p_{X \mid E}^{(t)}(y, x \mid e): y, x \in \mathcal{X}\right)$. Therefore, we see that the stationary distribution $\pi$ is expressed in terms of the stationary distribution $\boldsymbol{\pi}_{E}$ of the environmental process, the Palm distributions $\pi_{\mathrm{a}(e)}$, and the $q_{E}(e)$-potentials of the Markov chains with transition rate matrices $\boldsymbol{Q}_{X \mid E}(e), e \in \mathcal{E}$. From now on, we suppress the subscript ' $q(e)$ ' and write $m(y, x \mid e)$ instead of $m_{q(e)}(y, x \mid e), e \in \mathcal{E}$.

The $\alpha$-potentials play a very important role, both in the theory, and in the applications of Markov chains; Chapters 7 and 8 of Çinlar (1975) present the main results, and some applications. For a nice, recent account, see also Norris (1997, Chapter 4). For the sake of completeness, we state the following fundamental result about the computation of $\alpha$-potentials directly from the transition rate matrix of a Markov chain.

Proposition 1. Let $\boldsymbol{Q}$ be the transition rate matrix of a continuous-time Markov chain with transition probabilities $\left(p^{(t)}(x, y)\right)$ and $\alpha$-potentials

$$
m_{\alpha}(x, y)=\int_{0}^{\infty} \mathrm{e}^{-\alpha t} p^{(t)}(x, y) \mathrm{d} t, \quad \alpha>0 .
$$

Then, the matrix $\boldsymbol{M}_{\alpha}=\left(m_{\alpha}(x, y)\right)$ is the unique bounded solution of $(\alpha \boldsymbol{I}-\boldsymbol{Q}) \boldsymbol{X}=\boldsymbol{I}$ (or $\boldsymbol{X}(\alpha \boldsymbol{I}-\boldsymbol{Q})=\boldsymbol{I})$, $\boldsymbol{I}$ being the identity matrix. Moreover, when the state space of the Markov chain is finite, we find that $\boldsymbol{M}_{\alpha}=(\alpha \boldsymbol{I}-\boldsymbol{Q})^{-1}$.

Proof. See Norris (1997, Theorem 4.2.6).

By applying this proposition, we can compute the matrix $\boldsymbol{M}(e)=(m(y, x \mid e): y, x \in \mathcal{X})$ that we need for the inversion formula: it is the unique bounded solution of the matrix equations

$$
\begin{aligned}
& \left(q_{E}(e) \boldsymbol{I}-\boldsymbol{Q}_{X \mid E}(e)\right) \boldsymbol{X}=\boldsymbol{I}, \\
& \boldsymbol{X}\left(q_{E}(e) \boldsymbol{I}-\boldsymbol{Q}_{X \mid E}(e)\right)=\boldsymbol{I} .
\end{aligned}
$$

In the special case where $\boldsymbol{Q}_{X \mid E}(e)$ is finite dimensional, we find that

$$
\boldsymbol{M}(e)=\left(q_{E}(e) \boldsymbol{I}-\boldsymbol{Q}_{X \mid E}(e)\right)^{-1} .
$$

Therefore, the quantities $m(y, x \mid e)$ are the entries of the matrix $\left(q_{E}(e) \boldsymbol{I}-\boldsymbol{Q}_{X \mid E}(e)\right)^{-1}$ and can be computed by performing a simple matrix inversion. In many cases where the matrix $\boldsymbol{Q}_{X \mid E}(e)$ has a special structure, this inversion is carried out at a very low computational cost. Hence, we do not need to perform the laborious task of computing the transient probabilities $p_{X \mid E}^{(t)}(y, x \mid e)$. In Section 6, we will present several computations of $\boldsymbol{M}(e)$ in the framework of specific applications.

Remark 3. For a fixed $e \in \mathcal{E}$, to compute $\pi_{e}=(\pi(e, x): x \in \mathcal{X})$ when $\pi_{\mathrm{a}(e)}$ is given, we must compute the matrix $\boldsymbol{M}(e)=\left(q_{E}(e) \boldsymbol{I}-\boldsymbol{Q}_{X \mid E}(e)\right)^{-1}$ and then apply (20). This reveals the important fact that, given $\pi_{\mathrm{a}(e)}$, the only information that we need to compute $\boldsymbol{\pi}_{e}$ lies in $\pi_{E}(e), q_{E}(e)$, and $\boldsymbol{Q}_{X \mid E}(e)$. In particular, we do not need the matrices $\boldsymbol{Q}_{X \mid E}\left(e^{\prime}\right)$ for $e^{\prime} \neq e$. This is in some sense a decomposability result. From this perspective, the inversion formula can be considered to be a generalized product-form result. 
Another consequence of the inversion formula is that the original system of full balance equations (4) can be transformed into another system of equations that is more convenient in some cases. Indeed, by substituting $\pi_{\mathrm{a}(e)}(x)$, given by (5), into the inversion formula (15), we obtain

$$
\pi(e, x)=\sum_{e^{\prime} \neq e} \sum_{x^{\prime} \in \mathcal{X}} \pi\left(e^{\prime}, x^{\prime}\right) q_{E}\left(e^{\prime}, e\right) \sum_{y \in \mathcal{X}} \alpha\left(y \mid e^{\prime}, x^{\prime}, e\right) m(y, x \mid e), \quad e \in \mathcal{E}, x \in \mathcal{X} .
$$

Moreover, we have the following result.

Theorem 3. The system of equations (24) is equivalent to the system of full balance equations (4). Therefore, the unique solution of (24) that satisfies the normalization equation $\sum_{e \in \mathcal{E}} \sum_{x \in X} \pi(e, x)=1$ is the stationary distribution of $\{(E(t), X(t))\}$.

Proof. From the above discussion, we have already seen that the full balance equations (4) imply (24) via the inversion formula. Conversely, suppose that $(\pi(e, x))$ is a solution of $(24)$. Then, we have that

$$
\begin{aligned}
\pi(e, x) & \left(q_{E}(e)+\sum_{x^{\prime} \neq x} q_{X \mid E}\left(x, x^{\prime} \mid e\right)\right) \\
= & \sum_{e^{\prime} \neq e} \sum_{x^{\prime} \in \mathcal{X}} \pi\left(e^{\prime}, x^{\prime}\right) q_{E}\left(e^{\prime}, e\right) \\
& \times \sum_{y \in X} \alpha\left(y \mid e^{\prime}, x^{\prime}, e\right) m(y, x \mid e)\left(q_{E}(e)+\sum_{x^{\prime} \neq x} q_{X \mid E}\left(x, x^{\prime} \mid e\right)\right) .
\end{aligned}
$$

By (22), we have that $\boldsymbol{M}(e)\left(q_{E}(e) \boldsymbol{I}-\boldsymbol{Q}_{X \mid E}(e)\right)=\boldsymbol{I}$. By equating the $(y, x)$ entries of the left- and the right-hand sides, we obtain

$$
\begin{aligned}
& m(y, x \mid e)\left(q_{E}(e)+\sum_{x^{\prime} \neq x} q_{X \mid E}\left(x, x^{\prime} \mid e\right)\right) \\
& \quad-\sum_{z \neq x} m(y, z \mid e) q_{X \mid E}(z, x \mid e)=\mathbf{1}_{\{y\}}(x), \quad y, x \in \mathcal{X} .
\end{aligned}
$$

Therefore, (25) assumes the form

$$
\begin{aligned}
\pi(e, x) & \left(q_{E}(e)+\sum_{x^{\prime} \neq x} q_{X \mid E}\left(x, x^{\prime} \mid e\right)\right) \\
= & \sum_{e^{\prime} \neq e} \sum_{x^{\prime} \in \mathcal{X}} \pi\left(e^{\prime}, x^{\prime}\right) q_{E}\left(e^{\prime}, e\right) \alpha\left(x \mid e^{\prime}, x^{\prime}, e\right) \\
& +\sum_{z \neq x}\left(\sum_{e^{\prime} \neq e} \sum_{x^{\prime} \in \mathcal{X}} \pi\left(e^{\prime}, x^{\prime}\right) q_{E}\left(e^{\prime}, e\right) \sum_{y \in \mathcal{X}} \alpha\left(y \mid e^{\prime}, x^{\prime}, e\right) m(y, z \mid e)\right) q_{X \mid E}(z, x \mid e) .
\end{aligned}
$$

The multiple sum in brackets in the last term is equal to $\pi(e, z)$, because of (24). Therefore (27) is reduced to the full balance equations (4). 
When the triggering probability functions are of type 2 or 3 , we have, more specifically, the following corollary.

Corollary 3. Consider a model with transition rates given by (1) and a type-2 or -3 triggering probability function. Then, the stationary distribution $\pi$ is directly computable from $\pi_{E}$ and $\boldsymbol{M}(e), e \in \mathcal{E}$. For type-2 and -3 models, the stationary distributions are given respectively by

$$
\begin{aligned}
& \pi(e, x)=\sum_{e^{\prime} \neq e} \pi_{E}\left(e^{\prime}\right) q_{E}\left(e^{\prime}, e\right) \sum_{y \in \mathcal{X}} \gamma\left(y \mid e^{\prime}, e\right) m(y, x \mid e), \quad e \in \mathcal{E}, x \in \mathcal{X}, \\
& \pi(e, x)=\pi_{E}(e) q_{E}(e) \sum_{y \in \mathcal{X}} \beta(y \mid e) m(y, x \mid e), \quad e \in \mathcal{E}, x \in \mathcal{X} .
\end{aligned}
$$

Proof. By substituting $\alpha\left(y \mid e^{\prime}, x^{\prime}, e\right)=\gamma\left(y \mid e^{\prime}, e\right)$ or $\alpha\left(y \mid e^{\prime}, x^{\prime}, e\right)=\beta(y \mid e)$ into (24), we obtain (28) or (29), respectively.

Remark 4. Corollary 3 strengthens Remark 3 . We have a complete decomposability result for models with type-2 and -3 triggering probability functions: to compute $\pi_{e}=(\pi(e, x): x \in \mathcal{X})$, we need $\left(\pi_{E}(e): e \in \mathcal{E}\right), \boldsymbol{Q}_{E}$, and $\boldsymbol{M}(e)$. We do not need $\boldsymbol{M}\left(e^{\prime}\right)$ for $e^{\prime} \neq e$.

In the case of type-1 models, which are the most common in the literature, (24) assumes the form

$$
\pi(e, x)=\sum_{e^{\prime} \neq e} \sum_{y \in \mathcal{X}} \pi\left(e^{\prime}, y\right) q_{E}\left(e^{\prime}, e\right) m(y, x \mid e), \quad e \in \mathcal{E}, x \in \mathcal{X} .
$$

This formula may facilitate the exact computation of the stationary distribution $\pi$ in certain cases that we present later, in applications of the inversion formula in Section 6. As a trivial example of its use, consider the case where there are only two environmental states and $N$ states for the process $\{X(t)\}$. Then, by applying (30) twice, we obtain

$$
\pi(e, x)=q_{E}\left(e, e^{\prime}\right) q_{E}\left(e^{\prime}, e\right) \sum_{z \in \mathcal{X}} \pi(e, z) \sum_{y \in \mathcal{X}} m\left(z, y \mid e^{\prime}\right) m(y, x \mid e), \quad x \in \mathcal{X} .
$$

Therefore, the $2 N \times 2 N$ system of balance equations is reduced to an $N \times N$ system. In a similar manner, if the environmental process has $K$ states $e_{1}, e_{2}, \ldots, e_{K}$, which are always visited in the order $e_{1} \rightarrow e_{2} \rightarrow \cdots \rightarrow e_{K} \rightarrow e_{1}$, etc. (i.e. the only positive transition rates of the process are $\left.q_{E}\left(e_{1}, e_{2}\right), q_{E}\left(e_{2}, e_{3}\right), \ldots, q_{E}\left(e_{K-1}, e_{K}\right), q_{E}\left(e_{K}, e_{1}\right)\right)$, then the $K N \times K N$ system of balance equations is easily reduced to an $N \times N$ system. Formula (24) is also useful in more complicated cases, as we will see in Section 6.

The inversion formula (15), or its equivalent (20), is also useful in simulation studies of certain intractable models. In such cases, it is convenient to 'observe' the system only during environmental changes, and deduce its stationary distribution using the inversion formula.

\section{Applications of the product-form results}

In this section, we apply the results of Section 3 to several models occurring in queueing, and other applied probability problems. 


\subsection{Product-form stationary distribution for a Markov-modulated birth-death process}

A continuous-time Markov chain $\{(E(t), X(t))\}$ on $\mathcal{E} \times \mathbb{Z}_{+}$is called a Markov-modulated birth-death process if its nonzero transition rates have the form

$$
q\left((e, x),\left(e^{\prime}, x^{\prime}\right)\right)= \begin{cases}\lambda(x \mid e) & \text { if } e^{\prime}=e, x \geq 0, x^{\prime}=x+1 \\ \mu(x \mid e) & \text { if } e^{\prime}=e, x \geq 1, x^{\prime}=x-1, \\ q_{E}\left(e, e^{\prime}\right) & \text { if } e^{\prime} \neq e, x \geq 0, x^{\prime}=x\end{cases}
$$

where $\lambda(x \mid e)$ and $\mu(x \mid e)$ denote the birth and death rates, respectively.

Zhu (1994) proved that a sufficient condition for a product-form stationary distribution is for $\lambda(x-1 \mid e) / \mu(x \mid e)$ to be independent of $e$ for all $x \geq 1$. Using Corollary 1, we can immediately show that Zhu's condition is actually necessary and sufficient. More specifically, we have the following result.

Corollary 4. Let $\{(E(t), X(t))\}$ be a Markov-modulated birth-death process with transition rates given by (31), where, for every environmental state e, the corresponding birth-death process with birth rates $\lambda(x \mid e)$ and death rates $\mu(x \mid e)$ is positive recurrent. Then, the following statements are equivalent.

(i) $\lambda(x-1 \mid e) / \mu(x \mid e)$ is independent of e for all $x \geq 1$.

(ii) In stationarity, $E(t)$ and $X(t)$ are independent, that is, $\pi$ has the form (12).

Under conditions ( $i$ ) and (ii), $\pi$ is given by

$$
\pi(e, x)=\pi_{E}(e) B \prod_{i=1}^{x} \rho(i), \quad e \in \mathcal{E}, x \in \mathcal{X},
$$

where $\left(\pi_{E}(e): e \in \mathcal{E}\right)$ is the stationary distribution of the Markov chain with transition rates $\left(q_{E}\left(e, e^{\prime}\right)\right), \rho(i)=\lambda(i-1 \mid e) / \mu(i \mid e)(i \geq 1, e \in \mathcal{E})$, and $B=\left(1+\sum_{x=1}^{\infty} \prod_{i=1}^{x} \rho(i)\right)^{-1}$. Moreover, the reversed process $\left\{\left(E^{\mathrm{R}}(t), X^{\mathrm{R}}(t)\right)\right\}$ is a Markov-modulated birth-death process with nonzero transition rates

$$
q^{\mathrm{R}}\left((e, x),\left(e^{\prime}, x^{\prime}\right)\right)= \begin{cases}\lambda(x \mid e) & \text { if } e^{\prime}=e, x \geq 0, x^{\prime}=x+1, \\ \mu(x \mid e) & \text { if } e^{\prime}=e, x \geq 1, x^{\prime}=x-1, \\ \pi_{E}\left(e^{\prime}\right) q_{E}\left(e^{\prime}, e\right) / \pi_{E}(e) & \text { if } e^{\prime} \neq e, x \geq 0, x^{\prime}=x .\end{cases}
$$

Proof. The transition rates given by (31) have the form (1) with type-1 triggering probability functions $\alpha\left(x^{\prime} \mid e, x, e^{\prime}\right)=\mathbf{1}_{\{x\}}\left(x^{\prime}\right)$, and the matrices $\boldsymbol{Q}_{X \mid E}(e)(e \in \mathcal{E})$ correspond to birthdeath processes, i.e.

$$
q_{X \mid E}\left(x, x^{\prime} \mid e\right)= \begin{cases}\lambda(x \mid e) & \text { if } x \geq 0, x^{\prime}=x+1 \\ \mu(x \mid e) & \text { if } x \geq 1, x^{\prime}=x-1 \\ 0 & \text { otherwise. }\end{cases}
$$

Therefore, Corollary 1 is applicable and, hence, we have that condition (ii) holds if and only if all the stationary distributions $\pi_{X \mid E}(e)$ coincide. However,

$$
\pi_{X \mid E}(x \mid e)=c(e) \prod_{i=1}^{x} \frac{\lambda(i-1 \mid e)}{\mu(i \mid e)}, \quad x \in \mathcal{X}
$$


and we have that $\pi_{X \mid E}(e)$ coincide if and only if $\lambda(x-1 \mid e) / \mu(x \mid e)$ is independent of $e$ for $x \geq 1$. The rest of the corollary is obvious, in light of (9) and (13).

\subsection{Product-form stationary distribution for a Jackson network in a random environment}

A Jackson network in a random environment is a continuous-time Markov chain on $\varepsilon \times \mathbb{Z}_{+}^{J}$ with transition rates given by (1) and type-1 triggering probability functions and matrices $\boldsymbol{Q}_{X \mid E}(e)(e \in \mathcal{E})$ corresponding to Jackson networks, i.e.

$$
q_{X \mid E}\left(\boldsymbol{x}, \boldsymbol{x}^{\prime} \mid e\right)= \begin{cases}\lambda(e) p_{0 j}(e) & \text { if } \boldsymbol{x}^{\prime}=\boldsymbol{x}+\boldsymbol{e}_{j}, \\ \mu_{i}\left(x_{i} \mid e\right) p_{i j}(e) & \text { if } \boldsymbol{x}^{\prime}=\boldsymbol{x}-\boldsymbol{e}_{i}+\boldsymbol{e}_{j}, \\ \mu_{i}\left(x_{i} \mid e\right) p_{i 0}(e) & \text { if } \boldsymbol{x}^{\prime}=\boldsymbol{x}-\boldsymbol{e}_{i}, \\ 0 & \text { otherwise, }\end{cases}
$$

where $\lambda(e), \mu_{i}\left(x_{i} \mid e\right)$, and $p_{i j}(e)$ denote the arrival rate, the service rates, and the routing probabilities, respectively. Also, by $\boldsymbol{x}=\left(x_{1}, x_{2}, \ldots, x_{J}\right)$, we denote a generic state of the network representing the queue lengths at the $J$ stations, and $\boldsymbol{e}_{j}$ is the $j$ th unit vector with $J$ components (with 1 in the $j$ th position and 0 elsewhere).

Zhu (1994) and Tsitsiashvili et al. (2002) proved a sufficient condition for product form. Using Corollary 1, we can easily show both the necessity and the sufficiency of that condition.

Corollary 5. Let $\{(E(t), X(t))\}$ be a Jackson network in a random environment with transition rates given by (32) and, for every $e \in \mathcal{E}$, let $\alpha(e)=\left(\alpha_{1}(e), \alpha_{2}(e), \ldots, \alpha_{J}(e)\right)$ be the unique solution to the system of equations

$$
\alpha_{j}(e)=\lambda(e) p_{0 j}(e)+\sum_{i=1}^{J} \alpha_{i}(e) p_{i j}(e), \quad j=1,2, \ldots, J .
$$

Moreover, we assume that

$$
\sum_{x_{j}=1}^{\infty} \frac{\alpha_{j}(e)^{x_{j}}}{\mu_{j}(1 \mid e) \mu_{j}(2 \mid e) \cdots \mu_{j}\left(x_{j} \mid e\right)}<\infty, \quad e \in \mathcal{E} .
$$

The following statements are equivalent.

(i) $\alpha_{j}(e) / \mu_{j}\left(x_{j} \mid e\right)$ is independent of $e$.

(ii) In stationarity, $E(t)$ and $\boldsymbol{X}(t)$ are independent, that is, $\pi$ has the form (12).

Under conditions (i) and (ii), $\pi$ is given by

$$
\pi(e, \boldsymbol{x})=\pi_{E}(e) B \prod_{j=1}^{J} \prod_{i=1}^{x_{j}} \rho_{j}(i), \quad e \in \mathcal{E}, \boldsymbol{x} \in \mathcal{X}=\mathbb{Z}_{+}^{J},
$$

where $\left(\pi_{E}(e): e \in \mathcal{E}\right)$ is the stationary distribution of the Markov chain with transition rates $\left(q_{E}\left(e, e^{\prime}\right)\right), \rho_{j}(i)=\alpha_{j}(e) / \mu_{j}(i \mid e)(i \geq 1, j=1,2, \ldots, J, e \in \mathcal{E})$, and

$$
B=\left(\sum_{x_{1}, x_{2}, \ldots, x_{J}} \prod_{j=1}^{J} \prod_{i=1}^{x_{j}} \rho_{j}(i)\right)^{-1}
$$


Moreover, the reversed process $\left\{\left(E^{\mathrm{R}}(t), X^{\mathrm{R}}(t)\right)\right\}$ is a Jackson network in a random environment with parameters

$$
\begin{aligned}
q_{E}^{\mathrm{R}}\left(e, e^{\prime}\right) & =\pi_{E}\left(e^{\prime}\right) q_{E}\left(e^{\prime}, e\right) / \pi_{E}(e), \quad e \in \mathcal{E}, \\
\lambda^{\mathrm{R}}(e) p_{0 j}^{\mathrm{R}}(e) & =\alpha_{j}(e) p_{j 0}(e), \quad e \in \mathcal{E}, j=1,2, \ldots, J, \\
\mu_{j}^{\mathrm{R}}\left(x_{j} \mid e\right) & =\mu_{j}\left(x_{j} \mid e\right), \quad e \in \mathcal{E}, j=1,2, \ldots, J, x_{j} \geq 1, \\
p_{i j}^{\mathrm{R}}(e) & =\alpha_{j}(e) p_{j i}(e) / \alpha_{i}(e), \quad e \in \mathcal{E}, i, j=1,2, \ldots, J, \\
p_{i 0}^{\mathrm{R}}(e) & =\lambda(e) p_{0 i}(e) / \alpha_{i}(e), \quad e \in \mathcal{E}, i=1,2, \ldots, J .
\end{aligned}
$$

Proof. Using Corollary 1, we have that condition (ii) holds if and only if all the stationary distributions $\pi_{X \mid E}(e)$ coincide. However,

$$
\pi_{X \mid E}(\boldsymbol{x} \mid e)=c(e) \prod_{j=1}^{J} \prod_{i=1}^{x_{j}} \frac{\alpha_{j}(e)}{\mu_{j}(i \mid e)}, \quad \boldsymbol{x} \in \mathbb{Z}_{+}^{J},
$$

and we thus have that $\pi_{X \mid E}(e)$ coincide if $\alpha_{j}(e) / \mu_{j}\left(x_{j} \mid e\right)$ is independent of $e$. The rest of the corollary follows easily, using (9) and (13).

\subsection{Product-form stationary distribution for a heterogeneous blocking system in a ran- dom environment}

Fakinos (1982) considered a heterogeneous blocking system with a set of servers $C=$ $\{1,2, \ldots, c\}$. The rate of arrival when there are $i$ busy servers is $\lambda(i)$. If there are free servers at an arrival epoch, an arriving customer chooses one of them randomly, receives service, and leaves the system. If, on the other hand, all servers are busy, the customer leaves the system immediately. The service time of a customer assigned to server $s \in C$ is exponentially distributed with parameter $\mu_{s}$ (mean $1 / \mu_{s}$ ). The rate of service when there are $i$ busy servers is $r(i)$. This system will be referred to as the heterogeneous blocking system with parameters $\lambda(i), r(i)$, and $\mu_{s}$. The state of the system at time $t$ is the set of busy servers $X(t)$. The process $\{X(t)\}$ is a continuous-time Markov chain on the set $\mathcal{P}(C)$ of all subsets of $C$.

Fakinos (1982) showed that the stationary distribution $\left(\pi_{X}(x): x \in \mathcal{P}(C)\right)$ of this system is given by

$$
\pi_{X}(x)=G(c-|x|) ! \prod_{s \in x} \frac{1}{\mu_{s}} \prod_{i=1}^{|x|} \frac{\lambda(i-1)}{r(i)}, \quad x \in \mathcal{P}(C),
$$

where $|x|$ is the cardinality of the set $x$ and $G$ is a normalizing constant.

Falin (1996) considered this system in a random environment, i.e. he considered a continuous-time Markov chain on $\mathscr{E} \times \mathcal{P}(C)$ with transition rates given by (1) and type-1 triggering probability functions and matrices $\boldsymbol{Q}_{X \mid E}(e)$ corresponding to a heterogeneous blocking system with parameters $\lambda(i \mid e), r(i \mid e)$, and $\mu_{s}$, for every fixed environmental state $e$. He proved a necessary and sufficient condition for product form, which follows immediately, in light of Corollary 1 . We state this result below.

Corollary 6. Let $\{(E(t), X(t))\}$ be a heterogeneous blocking system in a random environment. The following statements are equivalent.

(i) $\lambda(i-1 \mid e) / r(i \mid e)$ is independent of e for all $i=1,2, \ldots, c$.

(ii) In stationarity, $E(t)$ and $X(t)$ are independent, that is, $\pi$ has the form (12). 
Under conditions ( $i$ ) and (ii), $\pi$ is given by

$$
\pi(e, x)=\pi_{E}(e) G(c-|x|) ! \prod_{s \in x} \frac{1}{\mu_{s}} \prod_{i=1}^{|x|} \rho(i), \quad e \in \mathcal{E}, x \in \mathcal{P}(C),
$$

where $\left(\pi_{E}(e): e \in \mathcal{E}\right)$ is the stationary distribution of a Markov chain with transition rates $\left(q_{E}\left(e, e^{\prime}\right)\right), \rho(i)=\lambda(i-1 \mid e) / r(i \mid e)(i=1,2, \ldots, c)$, and

$$
G=\left(\sum_{x \in \mathcal{P}(C)}(c-|x|) ! \prod_{s \in x} \frac{1}{\mu_{s}} \prod_{i=1}^{|x|} \rho(i)\right)^{-1} .
$$

Proof. Using Corollary 1, we have that condition (ii) holds if and only if all the stationary distributions $\boldsymbol{\pi}_{X \mid E}(e)$ coincide. However, by (33), we have

$$
\pi_{X \mid E}(x \mid e)=G(e)(c-|x|) ! \prod_{s \in x} \frac{1}{\mu_{s}} \prod_{i=1}^{|x|} \frac{\lambda(i-1 \mid e)}{r(i \mid e)}, \quad x \in \mathcal{P}(C),
$$

where $G(e)$ is the normalizing constant $G$ for the specific $e$ under consideration, and we have that $\pi_{X \mid E}(e)$ coincide if $\lambda(i-1 \mid e) / r(i \mid e)$ is independent of $e$ for all $i=1,2, \ldots, c$.

\section{Applications of the inversion formula}

In this section, we present several models that can be studied effectively using the results of Section 4. As we have already seen, the use of the inversion formula and its consequences (formulae (24), (28), (29), and (30)) requires the computation of the matrices $\boldsymbol{M}(e)=$ $(m(x, y \mid e): x, y \in \mathcal{X}), e \in \mathcal{E}$. In principle, these computations correspond to a matrix inversion, when $\mathcal{X}$ is finite (see formula (23)). In some remarkable cases where $\boldsymbol{Q}_{X \mid E}(e)$ has a special structure (for example, when it is (block) tridiagonal or (block) triangular), we can obtain explicit expressions at a very low computational cost. We begin by presenting a variety of such cases. For simplicity, we suppress the dependence on $e$ of the transition rates $q_{X \mid E}(y, x \mid e)$ in the presentation of the following lemmas. First, we consider the cases of pure-birth and pure-death processes.

Lemma 2. (i) For a pure-birth transition rate matrix $\boldsymbol{Q}_{X \mid E}(e)$ with birth rates $\lambda(y)$, the nonzero $q_{E}(e)$-potentials $m(y, x \mid e)$ are given by

$$
m(y, x \mid e)= \begin{cases}\frac{1}{q_{E}(e)+\lambda(y)} & \text { if } 0 \leq x=y, \\ \frac{1}{q_{E}(e)+\lambda(y)} \prod_{i=y+1}^{x} \frac{\lambda(i-1)}{q_{E}(e)+\lambda(i)} & \text { if } 0 \leq y<x .\end{cases}
$$

In particular, for a Poisson birth process with rate $\lambda$, we have

$$
m(y, x \mid e)=\frac{1}{q_{E}(e)+\lambda}\left(\frac{\lambda}{q_{E}(e)+\lambda}\right)^{x-y} \quad \text { if } 0 \leq y \leq x .
$$

The corresponding kth convolution of $m(y, x \mid e)$ is given by

$$
m^{(k)}(y, x \mid e)=\left(\begin{array}{c}
x-y+k-1 \\
k-1
\end{array}\right) \frac{\lambda^{x-y}}{\left(q_{E}(e)+\lambda\right)^{x-y+k}} \quad \text { if } 0 \leq y \leq x .
$$


(ii) For a pure-death transition matrix $\boldsymbol{Q}_{X \mid E}(e)$ with death rates $\mu(y)$, the nonzero $q_{E}(e)$ potentials $m(y, x \mid e)$ are given by

$$
m(y, x \mid e)= \begin{cases}\frac{1}{q_{E}(e)} & \text { if } 0=x=y, \\ \frac{1}{q_{E}(e)+\mu(y)} & \text { if } 1 \leq x=y, \\ \frac{1}{q_{E}(e)+\mu(y)} \prod_{i=x}^{y-1} \frac{\mu(i+1)}{q_{E}(e)+\mu(i)} & \text { if } 1 \leq x<y, \\ \frac{1}{q_{E}(e)+\mu(y)}\left(\prod_{i=1}^{y-1} \frac{\mu(i+1)}{q_{E}(e)+\mu(i)}\right) \frac{\mu(1)}{q_{E}(e)} & \text { if } 0=x<y .\end{cases}
$$

In particular, for a Poisson death process with rate $\mu$, we have

$$
m(y, x \mid e)= \begin{cases}\frac{1}{q_{E}(e)} & \text { if } 0=x=y, \\ \frac{1}{q_{E}(e)+\mu}\left(\frac{\mu}{q_{E}(e)+\mu}\right)^{y-x} & \text { if } 1 \leq x \leq y, \\ \frac{1}{q_{E}(e)+\mu}\left(\frac{\mu}{q_{E}(e)+\mu}\right)^{y-1} \frac{\mu}{q_{E}(e)} & \text { if } 0=x<y .\end{cases}
$$

Proof. In the case of a pure-birth transition rate matrix, (22) or, equivalently, (26) assumes the (collective) form

$$
\begin{aligned}
& m(y, x \mid e)\left(q_{E}(e)+\lambda(x)\right)=0 \quad \text { if } 0 \leq x<y, \\
& m(y, y \mid e)\left(q_{E}(e)+\lambda(y)\right)=1 \quad \text { if } 0 \leq x=y, \\
& m(y, x \mid e)\left(q_{E}(e)+\lambda(x)\right)=m(y, x-1 \mid e) \lambda(x-1) \quad \text { if } 0 \leq y<x .
\end{aligned}
$$

The first branch of $m(y, x \mid e)$ in (34) is now obvious by (39) and (40), while the second branch follows by iterating (41). In the case where $\lambda(y)=\lambda$, (34) is reduced to (35). For a fixed $y$, $\left(m(y, x \mid e) q_{E}(e): x=y, y+1, \ldots\right)$ is a geometric distribution. Therefore, its $k$ th convolution (i.e. $\left.\left(m^{(k)}(y, x \mid e)\left(q_{E}(e)\right)^{k}: x=y, y+1, \ldots\right)\right)$ is a negative binomial distribution and we can easily obtain (36). Statement (ii), for the pure-death case, can be proved similarly.

We now turn our attention to finite birth-death processes. Let $\boldsymbol{Q}_{X \mid E}(e)$ be the transition rate matrix of a finite birth-death process on $\mathcal{X}=\{0,1,2, \ldots, N\}$, with nonzero rates

$$
q_{X \mid E}(y, x \mid e)= \begin{cases}\lambda(y) & \text { if } 0 \leq y \leq N-1, x=y+1, \\ \mu(y) & \text { if } 1 \leq y \leq N, x=y-1\end{cases}
$$

The so-called Karlin-McGregor polynomials $Q_{n}(x), n=0,1,2, \ldots, N+1$, are defined recursively by the relations

$$
\begin{aligned}
Q_{0}(s) & =1, \quad Q_{1}(s)=(\lambda(0)-s) / \lambda(0), \\
Q_{n+1}(s) & =\left[(\lambda(n)+\mu(n)-s) Q_{n}(s)-\mu(n) Q_{n-1}(s)\right] / \lambda(n), \quad 1 \leq n \leq N-1, \\
Q_{N+1}(s) & =(\mu(N)-s) Q_{N}(s)-\mu(N) Q_{N-1}(s) .
\end{aligned}
$$


Karlin and McGregor (1965) introduced these polynomials to study the transient behaviour of birth-death processes. They showed that $Q_{N+1}(s)$ has $N+1$ distinct, real zeros $s_{0}=0<$ $s_{1}<s_{2}<\cdots<s_{N}$. Moreover, the polynomials $Q_{n}(s)(n=0,1,2, \ldots, N)$ are orthogonal on the set $\left\{s_{0}, s_{1}, s_{2}, \ldots, s_{N}\right\}$ with respect to the positive weights $\rho_{0}, \rho_{1}, \rho_{2}, \ldots, \rho_{N}$, that is,

$$
\sum_{k=0}^{N} Q_{y}\left(s_{k}\right) Q_{x}\left(s_{k}\right) \rho_{k}=0, \quad 0 \leq y \neq x \leq N,
$$

where $\rho_{k}=\left(\sum_{i=0}^{N} Q_{i}^{2}\left(s_{k}\right) \psi_{i}\right)^{-1}(k=0,1,2, \ldots, N)$, and $\psi_{i}$ are the so-called potential coefficients, defined by

$$
\psi_{0}=1, \quad \psi_{n}=\frac{\lambda(0) \lambda(1) \cdots \lambda(n-1)}{\mu(1) \mu(2) \cdots \mu(n)}, \quad n=1,2, \ldots, N .
$$

The transition probabilities $p_{X \mid E}^{(t)}(y, x \mid e)$ then have the spectral representation

$$
p_{X \mid E}^{(t)}(y, x \mid e)=\psi_{x} \sum_{k=0}^{N} \mathrm{e}^{-s_{k} t} Q_{y}\left(s_{k}\right) Q_{x}\left(s_{k}\right) \rho_{k}, \quad y, x=0,1, \ldots, N .
$$

Using the Karlin-McGregor spectral representation (43) and the definition (21) of the $\alpha$-potential, we can immediately obtain the form of the $q_{E}(e)$-potentials in the case of finite birth-death processes. In fact, we have the following lemma.

Lemma 3. For a finite birth-death transition matrix $\boldsymbol{Q}_{X \mid E}(e)$ with transition rates given by (42), the $q_{E}(e)$-potentials $m(y, x \mid e)$ are given by

$$
m(y, x \mid e)=\psi_{x} \sum_{k=0}^{N} \frac{Q_{y}\left(s_{k}\right) Q_{x}\left(s_{k}\right) \rho_{k}}{s_{k}+q_{E}(e)}, \quad y, x=0,1, \ldots, N .
$$

In view of Lemma 3 , the problem of finding the $q_{E}(e)$-potentials $m(y, x \mid e)$, which we need in order to apply the inversion formula (and its consequences), reduces to that of finding the orthogonal polynomials $Q_{n}(s), n=0,1,2, \ldots, N$ and the roots $s_{0}, s_{1}, s_{2}, \ldots, s_{N}$ of $Q_{N+1}(s)$. In many cases, the birth-death polynomials are connected to classical orthogonal polynomials for which the form and the roots are well known. We present two important cases below.

Proposition 2. (i) For the finite birth-death transition matrix $\boldsymbol{Q}_{X \mid E}($ e), with nonzero transition rates

$$
q_{X \mid E}(y, x \mid e)= \begin{cases}\lambda & \text { if } 0 \leq y \leq N-1, x=y+1 \\ \mu & \text { if } 1 \leq y \leq N, x=y-1\end{cases}
$$

that corresponds to the M/M/I/N queue, the Karlin-McGregor polynomials $Q_{n}(x), n=$ $0,1,2, \ldots, N$, are given by

$$
Q_{n}(s)=\left(\frac{\mu}{\lambda}\right)^{n / 2}\left(U_{n}\left(\frac{\lambda+\mu-s}{2 \sqrt{\lambda \mu}}\right)-\sqrt{\frac{\mu}{\lambda}} U_{n-1}\left(\frac{\lambda+\mu-s}{2 \sqrt{\lambda \mu}}\right)\right),
$$

where $U_{n}(s)$ are the Chebyshev polynomials of the second kind. These polynomials are defined recursively by

$$
U_{-1}(s)=0, \quad U_{0}(s)=1, \quad U_{n+1}(s)=2 s U_{n}(s)-U_{n-1}(s), \quad n=0,1,2, \ldots
$$

The set of roots of $Q_{N+1}(s)$ is $\left\{0,\left\{\lambda+\mu-2(\lambda \mu)^{1 / 2} \cos (k \pi /(N+1)): k=1,2, \ldots, N\right\}\right\}$. 
(ii) For the finite birth-death transition matrix $\boldsymbol{Q}_{X \mid E}(e)$, with nonzero transition rates

$$
q_{X \mid E}(y, x \mid e)= \begin{cases}(N-y) \lambda & \text { if } 0 \leq y \leq N-1, x=y+1 \\ y \mu & \text { if } 1 \leq y \leq N, x=y-1\end{cases}
$$

that corresponds to the Engset model (an M/M/N queue with a finite number of potential customers $N$ ), the Karlin-McGregor polynomials $Q_{n}(x), n=0,1,2, \ldots, N$, are given by

$$
Q_{n}(s)=K_{n}\left(\frac{s}{\lambda+\mu} ; \frac{\lambda}{\lambda+\mu}, N\right),
$$

where $K_{n}(s ; p, N)$ are the Krawtchouk polynomials. These polynomials are defined recursively, for $N \in \mathbb{Z}_{+}$and $p \in(0,1)$, by

$$
\begin{aligned}
K_{-1}(s ; p, N) & =0, \quad K_{0}(s ; p, N)=1, \\
K_{n+1}(s ; p, N) & =\frac{(p(N-n)+n(1-p)-s) K_{n}(s ; p, N)-n(1-p) K_{n-1}(s ; p, N)}{p(N-n)},
\end{aligned}
$$

where $n=0,1,2, \ldots, N$. The set of roots of $Q_{N+1}(s)$ is $\{0,\{k(\lambda+\mu): k=1,2, \ldots, N\}\}$.

Proof. See van Assche et al. (1999).

We can now use the results of Section 4 to study several models.

\subsection{A population process subject to total catastrophes}

Consider a population that evolves according to some continuous-time Markov chain (e.g. a general immigration-birth-death process). Total catastrophes occur according to a renewal process and reduce the population size instantaneously to 0 . By approximating the interrenewal distribution by a phase-type distribution, we can see that this situation fits exactly within the framework of a continuous-time Markov chain in a random environment. To be exact, suppose that the natural evolution of the population follows the dynamics of a continuous-time Markov chain on $\mathcal{X}=\{0,1, \ldots, N\}$ or $\mathbb{Z}_{+}$, with rates $q_{X}\left(x, x^{\prime}\right)$, and that the catastrophes occur according to a renewal process with interrenewal distribution $\operatorname{Erlang}(K, v)$ (i.e. the sum of $K$ independent exponentials with parameter $v)$. If $X(t)$ is the population size at time $t$ and $E(t)$ is the number of the remaining $\operatorname{Exp}(v)$ phases until the next catastrophe, then $\{(E(t), X(t))\}$ is a continuous-time Markov chain on $\{1,2, \ldots, K\} \times \mathcal{X}$ with nonzero transition rates

$$
q\left((e, x),\left(e^{\prime}, x^{\prime}\right)\right)= \begin{cases}v & \text { if } e=2,3, \ldots, K, e^{\prime}=e-1, x^{\prime}=x \geq 0, \\ v & \text { if } e=1, e^{\prime}=K, x \geq 0, x^{\prime}=0, \\ q_{X}\left(x, x^{\prime}\right) & \text { if } e=1,2, \ldots, K, e^{\prime}=e, x \geq 0, x^{\prime} \neq x .\end{cases}
$$

We can use Theorem 3 to obtain the stationary distribution of this model.

Theorem 4. Let $\{(E(t), X(t))\}$ be a continuous-time Markov chain, with transition rates (44), which describes the evolution of a population subject to total catastrophes occurring according to a renewal process with Erlang interrenewal intervals. The stationary distribution of $\{(E(t), X(t))\}$ is given by

$$
\pi(e, x)=\frac{1}{K} \nu^{K-e+1} m^{(K-e+1)}(0, x), \quad e=1,2, \ldots, K, x \in \mathcal{X},
$$


where $m^{(k)}(y, x)$ is the kth convolution of the v-potentials of the process with rates $q_{X}\left(x, x^{\prime}\right)$. When the natural evolution of the population follows a Poisson process at rate $\lambda\left(i . e . q_{X}\left(x, x^{\prime}\right)=\right.$ $\left.\lambda \mathbf{1}_{\{x+1\}}\left(x^{\prime}\right)\right)$ we find that

$$
\pi(e, x)=\frac{1}{K}\left(\begin{array}{c}
x+K-e \\
K-e
\end{array}\right) \frac{v^{K-e+1} \lambda^{x}}{(v+\lambda)^{K-e+x+1}}, \quad e=1,2, \ldots, K, x \geq 0 .
$$

Proof. We are in the framework of (1), with nonzero parameters

$$
\begin{aligned}
q_{X \mid E}\left(x, x^{\prime} \mid e\right) & =q_{X}\left(x, x^{\prime}\right), \quad x^{\prime} \neq x, e=1,2, \ldots, K, \\
q_{E}\left(e, e^{\prime}\right) & = \begin{cases}v & \text { if } e=2,3, \ldots, K, e^{\prime}=e-1, \\
v & \text { if } e=1, e^{\prime}=K,\end{cases} \\
\alpha\left(x^{\prime} \mid e, x, e^{\prime}\right) & = \begin{cases}1 & \text { if } e=2,3, \ldots, K, e^{\prime}=e-1, x^{\prime}=x, \\
1 & \text { if } e=1, e^{\prime}=K, x \in \mathcal{X}, x^{\prime}=0 .\end{cases}
\end{aligned}
$$

In this case, (24) assumes the form

$$
\begin{aligned}
& \pi(e, x)=\sum_{x^{\prime}} \pi\left(e+1, x^{\prime}\right) \operatorname{vm}\left(x^{\prime}, x \mid e\right), \quad e=1,2, \ldots, K-1, x \in \mathcal{X}, \\
& \pi(K, x)=\sum_{x^{\prime}} \pi\left(1, x^{\prime}\right) \operatorname{vm}(0, x \mid K), \quad x \in \mathcal{X} .
\end{aligned}
$$

Equation (47) implies that $\pi_{E}(e)=1 / K(e=1,2, \ldots, K)$ while, by (49), we obtain $\pi(K, x)=v m(0, x \mid K) / K$. Then, by iterating (48), we obtain (45). In the special case of a Poisson process, (36) implies (46).

\subsection{An inventory process in a random environment}

Consider a storage space for a certain product that operates in a random environment. The random environment may represent the market conditions, the seasonal periodicity in the demand rate and/or the frequency of the inventory inspector visits. The random environment is described by a continuous-time Markov chain $\{(E(t))\}$ with state space $\mathcal{E}$ and transition rates $q_{E}\left(e, e^{\prime}\right)$. The manager of the storage space inspects and controls the inventory of the product each time an environmental change occurs. More specifically, when a transition $e \rightarrow e^{\prime}$ occurs, the inventory is forced to be $S\left(e, e^{\prime}\right)$ just after the transition (i.e. the inventory control is immediate). Whenever the environment is in state $e$, demands for the product arrive according to a Poisson process at rate $\mu_{e}$. Every demand is satisfied immediately and reduces the inventory level by one unit. If $X(t)$ is the inventory level at time $t$ then $\{(E(t), X(t))\}$ is a continuous-time Markov chain on $\mathscr{E} \times \mathbb{Z}_{+}$with nonzero transition rates

$$
q\left((e, x),\left(e^{\prime}, x^{\prime}\right)\right)= \begin{cases}\mu_{e} & \text { if } e \in \mathcal{E}, e^{\prime}=e, x \geq 1, x^{\prime}=x-1, \\ q_{E}\left(e, e^{\prime}\right) & \text { if } e \in \mathcal{E}, e^{\prime} \neq e, x \geq 0, x^{\prime}=S\left(e, e^{\prime}\right) .\end{cases}
$$

We can use Corollary 3 to obtain the stationary distribution of this model.

Theorem 5. Let $\{(E(t), X(t))\}$ be a continuous-time Markov chain with transition rates $(50)$, which describes the evolution of an inventory process in a random environment. The stationary 
distribution of $\{(E(t), X(t))\}$ is given by

$$
\pi(e, x)= \begin{cases}\sum_{\left\{e^{\prime} \neq e: S\left(e^{\prime}, e\right) \geq x\right\}} \frac{\pi_{E}\left(e^{\prime}\right) q_{E}\left(e^{\prime}, e\right)}{q_{E}(e)+\mu_{e}}\left(\frac{\mu_{e}}{q_{E}(e)+\mu_{e}}\right)^{S\left(e^{\prime}, e\right)-x} & \text { if } x \geq 1, \\ \sum_{e^{\prime} \neq e} \frac{\pi_{E}\left(e^{\prime}\right) q_{E}\left(e^{\prime}, e\right)}{q_{E}(e)+\mu_{e}}\left(\frac{\mu_{e}}{q_{E}(e)+\mu_{e}}\right)^{S\left(e^{\prime}, e\right)-1} \frac{\mu_{e}}{q_{E}(e)} & \text { if } x=0 .\end{cases}
$$

Proof. We are in the framework of the transition rates (1), with

$$
\begin{aligned}
& q_{X \mid E}\left(x, x^{\prime} \mid e\right)=\mu_{e}, \quad x \geq 1, x^{\prime}=x-1, e \in \mathcal{E}, \\
& \alpha\left(x^{\prime} \mid e, x, e^{\prime}\right)=\gamma\left(x^{\prime} \mid e, e^{\prime}\right)=\mathbf{1}_{\left\{S\left(e, e^{\prime}\right)\right\}}\left(x^{\prime}\right) .
\end{aligned}
$$

Using Corollary 3 and substituting the potentials $m(y, x \mid e)$ given by (38), we immediately obtain (51).

As another example, consider the case of an inventory process under the following ' $S$-policy'. The inventory is inspected periodically according to a renewal process with $\operatorname{Erlang}(K, v)$ interrenewal distribution, and replenished up to level $S$. The demands arrive according to a Poisson process at rate $\mu$. Let $E(t)$ be the number of the remaining $\operatorname{Exp}(v)$ phases until the next inspection, and let $X(t)$ be the inventory level at time $t$. In this case, $\{(E(t), X(t))\}$ is a continuous-time Markov chain on $\{1,2, \ldots, K\} \times\{0,1, \ldots, S\}$ with nonzero transition rates

$$
q\left((e, x),\left(e^{\prime}, x^{\prime}\right)\right)= \begin{cases}v & \text { if } e=2,3, \ldots, K, e^{\prime}=e-1, x \geq 0, x^{\prime}=x, \\ v & \text { if } e=1, e^{\prime}=K, x \geq 0, x^{\prime}=S, \\ \mu & \text { if } e=1,2, \ldots, K, e^{\prime}=e, x \geq 1, x^{\prime}=x-1 .\end{cases}
$$

We have the following theorem.

Theorem 6. Let $\{(E(t), X(t))\}$ be a continuous-time Markov chain with transition rates (52), describing a periodic review inventory model with $\operatorname{Erlang}(K, v)$ distribution, operating according to the above $S$-policy. The stationary distribution of $\{(E(t), X(t))\}$ can be obtained recursively from the formulae

$$
\pi(K, x)= \begin{cases}\frac{v}{K} \frac{1}{v+\mu}\left(\frac{\mu}{v+\mu}\right)^{S-x} & \text { if } 1 \leq x \leq S, \\ \frac{v}{K} \frac{1}{v+\mu}\left(\frac{\mu}{v+\mu}\right)^{S-1} \frac{\mu}{v} & \text { if } x=0,\end{cases}
$$

and, for $e=K-1, K-2, \ldots, 1$,

$$
\pi(e, x)= \begin{cases}\sum_{x^{\prime}=x}^{S} \pi\left(e+1, x^{\prime}\right) \frac{v}{v+\mu}\left(\frac{\mu}{v+\mu}\right)^{x^{\prime}-x} & \text { if } 1 \leq x \leq S, \\ \sum_{x^{\prime}=0}^{S} \pi\left(e+1, x^{\prime}\right) \frac{v}{v+\mu}\left(\frac{\mu}{v+\mu}\right)^{x^{\prime}-1} \frac{\mu}{v} & \text { if } x=0 .\end{cases}
$$


Proof. We are in the framework of the transition rates (1) with nonzero rates and triggering probabilities

$$
\begin{aligned}
& q_{X \mid E}\left(x, x^{\prime} \mid e\right)=\mu \quad \text { if } x \geq 1, x^{\prime}=x-1, \\
& q_{E}\left(e, e^{\prime}\right)= \begin{cases}v & \text { if } e=2,3, \ldots, K, e^{\prime}=e-1, \\
v & \text { if } e=1, e^{\prime}=K\end{cases} \\
& \alpha\left(x^{\prime} \mid e, x, e^{\prime}\right)= \begin{cases}1 & \text { if } e=2,3, \ldots, K, e^{\prime}=e-1, x^{\prime}=x, \\
1 & \text { if } e=1, e^{\prime}=K, 0 \leq x \leq S, x^{\prime}=S\end{cases}
\end{aligned}
$$

In this case, (24) and (38) imply (53) and (54).

\subsection{The $\mathrm{PH} / \mathrm{M}(n) / 1$ queue}

In a $\mathrm{PH} / \mathrm{M}(n) / 1$ queue, customers arrive according to a renewal process with phase-type interrenewal distribution. The customers have exponential, independent, and identically distributed service requirements, also independent of the arrival process. There exists one server, which works at a rate that depends on the number of customers present. Here, the process $\{(E(t))\}$ represents the remaining phases of the interrenewal distribution until the next arrival, while the process $\{(X(t))\}$ records the current number of customers. For simplicity and to be exact, we assume that the interarrival distribution is $\operatorname{Erlang}(K, v)$, that the service requirements are exponentially distributed with parameter 1 , and that the server works at rate $\mu(x)$ whenever there are $x$ customers. This is an $\mathrm{E}_{K} / \mathrm{M}(n) / 1$ queue. The process $\{(E(t), X(t))\}$ is a continuous-time Markov chain on $\{1,2, \ldots, K\} \times \mathbb{Z}_{+}$with nonzero transition rates

$$
q\left((e, x),\left(e^{\prime}, x^{\prime}\right)\right)= \begin{cases}v & \text { if } e=2,3, \ldots, K, e^{\prime}=e-1, x^{\prime}=x \geq 0 \\ v & \text { if } e=1, e^{\prime}=K, x \geq 0, x^{\prime}=x+1, \\ \mu(x) & \text { if } e=1,2, \ldots, K, e=e^{\prime}, x \geq 1, x^{\prime}=x-1\end{cases}
$$

We can use Theorem 3 to obtain the stationary distribution of this model.

Theorem 7. Let $\{(E(t), X(t))\}$ be a continuous-time Markov chain, with transition rates (55) and stationary distribution $(\pi(e, x))$, which describes the evolution of an $E_{K} / M(n) / 1$ queue. Also, let $m^{(k)}(y, x)$ be the kth convolution of the v-potentials $m(y, x)$ given by (37). Then, the stationary probabilities $\left(\pi(1, x): x \in \mathbb{Z}_{+}\right)$are the unique solution to the system of equations

$$
\begin{aligned}
\pi(1, x) & =v^{K} \sum_{x^{\prime}} \pi\left(1, x^{\prime}\right) m^{(K)}\left(x^{\prime}+1, x\right), \quad x \in \mathbb{Z}_{+}, \\
\sum_{x} \pi(1, x) & =1 / K .
\end{aligned}
$$

The stationary probabilities $\left(\pi(e, x): x \in \mathbb{Z}_{+}, e=2,3, \ldots, K\right)$ are given by

$$
\pi(e, x)=v^{K-e+1} \sum_{x^{\prime}} \pi\left(1, x^{\prime}\right) m^{(K-e+1)}\left(x^{\prime}+1, x\right), \quad x \in \mathbb{Z}_{+}, 2 \leq e \leq K .
$$


Proof. We are in the framework of (1) with nonzero rates and triggering probabilities

$$
\begin{aligned}
q_{X \mid E}\left(x, x^{\prime} \mid e\right) & =\mu(x) \quad \text { if } x \geq 1, x^{\prime}=x-1, \\
q_{E}\left(e, e^{\prime}\right) & = \begin{cases}v & \text { if } e=2,3, \ldots, K, e^{\prime}=e-1, \\
v & \text { if } e=1, e^{\prime}=K,\end{cases} \\
\alpha\left(x^{\prime} \mid e, x, e^{\prime}\right) & = \begin{cases}1 & \text { if } e=2,3, \ldots, K, e^{\prime}=e-1, x^{\prime}=x, \\
1 & \text { if } e=1, e^{\prime}=K, x \geq 0, x^{\prime}=x+1 .\end{cases}
\end{aligned}
$$

In this case, (24) assumes the form

$$
\begin{aligned}
& \pi(e, x)=\sum_{x^{\prime}} \pi\left(e+1, x^{\prime}\right) \nu m\left(x^{\prime}, x \mid e\right), \quad e=1,2, \ldots, K-1, x \geq 0, \\
& \pi(K, x)=\sum_{x^{\prime}} \pi\left(1, x^{\prime}\right) \nu m\left(x^{\prime}+1, x \mid e\right), \quad x \geq 0 .
\end{aligned}
$$

Equation (59) implies that $\pi_{E}(e)=1 / K(e=1,2, \ldots, K)$ and, specifically, results in (57). By iterating (60), we obtain

$$
\pi(1, x)=v^{K-1} \sum_{x^{\prime}} \pi\left(K, x^{\prime}\right) m^{(K-1)}\left(x^{\prime}, x\right) .
$$

Then, using (61), we obtain (56). Thus, the vector of the stationary probabilities $(\pi(1, x)$ : $x \in \mathbb{Z}_{+}$) is a solution to the system of equations (56), (57). Given such a solution, we define $\left(\pi(e, x): x \in \mathbb{Z}_{+}, e=2,3, \ldots, K\right)$ by (58). Then, it is easy to see that (56) and (58) imply (60) and (61). Moreover, (58) implies that

$$
\sum_{x} \pi(e, x)=v^{K-e+1} \sum_{x^{\prime}} \pi\left(1, x^{\prime}\right) \sum_{x} m^{(K-e+1)}\left(x^{\prime}+1, x\right), \quad e=2,3, \ldots, K .
$$

However, by the definition (21) of the $q_{E}(e)$-potentials, we can easily show that

$$
\sum_{x} m^{(k)}(y, x \mid e)=1 / q_{E}(e)^{k}
$$

and, hence,

$$
\sum_{x} m^{(K-e+1)}\left(x^{\prime}+1, x\right)=1 / v^{K-e+1} .
$$

Furthermore, (62) is reduced to $\sum_{x} \pi(e, x)=\sum_{x^{\prime}} \pi\left(1, x^{\prime}\right)=1 / K, e=2,3, \ldots, K$, by (57). Hence, $\sum_{e} \sum_{x} \pi(e, x)=1$, and we have that $\left(\pi(e, x): x \in \mathbb{Z}_{+}, e=1,2, \ldots, K\right)$ is a solution to the system $(60),(61)$ that also satisfies the normalization equation. By Theorem 3 , we then have that $(\pi(e, x))$, defined by (56)-(58), is the stationary distribution of $\{(E(t), X(t))\}$.

\subsection{The $M(n) / M(n) / 1 / N$ queue with a deteriorating server}

In an $\mathrm{M}(n) / \mathrm{M}(n) / 1 / N$ queue with a deteriorating server, there exists one server and a waiting room for $N$ customers (including the one in service). The server may be in one of $K$ operating modes $1,2, \ldots, K$ ( $K$ and 1 denoting the best and worst operating conditions, respectively) or in the nonoperating mode 0 (under repair). The server operates at mode $e$ for an $\operatorname{Exp}\left(v_{e}\right)$ time and then deteriorates, going to the next-worse mode $e-1$, etc. When the server reaches 
the nonoperating mode 0 , it goes to a repair facility. The repair times are $\operatorname{Exp}\left(v_{0}\right)$ random variables. After a repair, the server is reduced to the perfect operating condition $K$ and starts a new operation cycle.

When there are $x$ customers in the system and the state of the server is $e$, customers arrive to the system at exponential rate $\lambda(x \mid e)$, and the service rate is $\mu(x \mid e)$. We suppose that $\lambda(N \mid e)=0$ (no arrivals are accepted when the system is full) and that $\mu(x \mid 0)=0$ (no service is provided when the server is under repair). If $E(t)$ and $X(t)$ respectively denote the state of the server and the number of customers in the system at time $t$, then the process $\{(E(t), X(t))\}$ is a continuous-time Markov chain on $\{0,1,2, \ldots, K\} \times\{0,1, \ldots, N\}$ with nonzero transition rates

$$
q\left((e, x),\left(e^{\prime}, x^{\prime}\right)\right)= \begin{cases}v_{e} & \text { if } e=1,2, \ldots, K, e^{\prime}=e-1,0 \leq x \leq N, x^{\prime}=x, \\ v_{0} & \text { if } e=0, e^{\prime}=K, 0 \leq x \leq N, x^{\prime}=x, \\ \lambda(x \mid e) & \text { if } e=0,1, \ldots, K, e^{\prime}=e, 0 \leq x \leq N-1, x^{\prime}=x+1, \\ \mu(x \mid e) & \text { if } e=0,1, \ldots, K, e^{\prime}=e, 1 \leq x \leq N, x^{\prime}=x-1 .\end{cases}
$$

Again, we can use Theorem 3 to study the stationary distribution of this model.

Theorem 8. Let $\{(E(t), X(t))\}$ be a continuous-time Markov chain, with transition rates (63) and stationary distribution $(\pi(e, x))$, which describes the evolution of an $M(n) / M(n) / 1 / N$ queue with a deteriorating server. Also, let

$$
m^{(k)}\left(y, x \mid e_{1}, \ldots, e_{k}\right)=\sum_{x_{1}} \sum_{x_{2}} \cdots \sum_{x_{k-1}} m\left(y, x_{1} \mid e_{1}\right) m\left(x_{1}, x_{2} \mid e_{2}\right) \cdots m\left(x_{k-1}, x \mid e_{k}\right)
$$

be the kth convolution of the potentials $m\left(y, x \mid e_{1}\right), m\left(y, x \mid e_{2}\right), \ldots, m\left(y, x \mid e_{k}\right)$. Then, the stationary probabilities $(\pi(0, x): 0 \leq x \leq N)$ are the unique solution to the system of equations

$$
\begin{aligned}
\pi(0, x) & =\prod_{e^{\prime}=0}^{K} v_{e^{\prime}} \sum_{x^{\prime}=0}^{N} \pi\left(0, x^{\prime}\right) m^{(K+1)}\left(x^{\prime}, x \mid K, \ldots, 1,0\right), \quad 0 \leq x \leq N, \\
\sum_{x=0}^{K} \pi(0, x) & =\frac{1}{v_{0}}\left(\sum_{e^{\prime}=0}^{K} \frac{1}{v_{e^{\prime}}}\right)^{-1} .
\end{aligned}
$$

The stationary probabilities $(\pi(e, x): 1 \leq e \leq K, 0 \leq x \leq N)$ are given by

$$
\pi(e, x)=v_{0} \prod_{e^{\prime}=e+1}^{K} v_{e^{\prime}} \sum_{x^{\prime}=0}^{N} \pi\left(0, x^{\prime}\right) m^{(K-e+1)}\left(x^{\prime}, x \mid K, K-1, \ldots, e\right) .
$$

Proof. We are in the framework of (1) with type-1 triggering probability functions

$$
\alpha\left(x^{\prime} \mid e, x, e^{\prime}\right)=\mathbf{1}_{\{x\}}\left(x^{\prime}\right)
$$

and nonzero rates

$$
\begin{aligned}
q_{X \mid E}\left(x, x^{\prime} \mid e\right) & = \begin{cases}\lambda(x \mid e) & \text { if } 0 \leq x \leq N-1, x^{\prime}=x+1, \\
\mu(x \mid e) & \text { if } 1 \leq x \leq N, x^{\prime}=x-1,\end{cases} \\
q_{E}\left(e, e^{\prime}\right) & = \begin{cases}v_{e} & \text { if } e=1,2, \ldots, K, e^{\prime}=e-1, \\
v_{0} & \text { if } e=0, e^{\prime}=K .\end{cases}
\end{aligned}
$$


In this case, (24) assumes the form

$$
\begin{aligned}
& \pi(e, x)=\sum_{y} \pi(e+1, y) v_{e+1} m(y, x \mid e), \quad 0 \leq e \leq K-1,0 \leq x \leq N, \\
& \pi(K, x)=\sum_{y} \pi(0, y) v_{0} m(y, x \mid K), \quad 0 \leq x \leq N .
\end{aligned}
$$

By (67), we can easily show that

$$
\pi_{E}(e)=\frac{1}{v_{e}}\left(\sum_{e^{\prime}=0}^{K} \frac{1}{v_{e^{\prime}}}\right)^{-1}, \quad e=0,1, \ldots, K,
$$

and iterating (68) results in

$$
\pi(0, x)=\prod_{e^{\prime}=1}^{K} v_{e^{\prime}} \sum_{z} \pi(K, z) m^{(K)}(z, x \mid K-1, K-2, \ldots, 1,0) .
$$

Using (69), we obtain (64). Therefore, the vector of stationary probabilities $(\pi(0, x): 0 \leq x \leq$ $N)$ is a solution to the system (64), (65). We will prove that it also the unique solution to this system. To this end, consider a solution $(\pi(0, x): 0 \leq x \leq N)$ to the system (64), (65) and define $(\pi(e, x): 1 \leq e \leq K, 0 \leq x \leq N)$ according to (66). Then, we can easily see that (64) and (66) imply (68) and (69). Moreover, for every $e=1,2, \ldots, K$, by summing (66) for $0 \leq x \leq N$ we have

$$
\sum_{x=0}^{N} \pi(e, x)=v_{0} \prod_{e^{\prime}=e+1}^{K} v_{e^{\prime}} \sum_{x^{\prime}=0}^{N} \pi\left(0, x^{\prime}\right) \sum_{x=0}^{N} m^{(K-e+1)}\left(x^{\prime}, x \mid K, K-1, \ldots, e\right) .
$$

Using (21), we can show that $\sum_{x} m^{(k)}\left(y, x \mid e_{1}, e_{2}, \ldots, e_{k}\right)=\prod_{i=1}^{k}\left(1 / q_{E}\left(e_{i}\right)\right)$. In this case, we have $\sum_{x=0}^{N} m^{(K-e+1)}\left(x^{\prime}, x \mid K, K-1, \ldots, e\right)=\prod_{e^{\prime}=e}^{K}\left(1 / v_{e^{\prime}}\right)$ and (70) assumes the form

$$
\sum_{x=0}^{N} \pi(e, x)=\frac{\nu_{0}}{v_{e}} \sum_{x^{\prime}=0}^{N} \pi\left(0, x^{\prime}\right)=\frac{1}{v_{e}}\left(\sum_{e^{\prime}=0}^{K} \frac{1}{v_{e^{\prime}}}\right)^{-1}, \quad e=1,2, \ldots, K,
$$

where the second equality follows from (65). Hence, $\sum_{e=0}^{K} \sum_{x=0}^{N} \pi(e, x)=1$ and we conclude that $(\pi(e, x): 0 \leq e \leq K, 0 \leq x \leq N)$ is a solution to the system (68), (69) that also satisfies the normalization equation. By Theorem 3 , we then have that $(\pi(e, x))$, defined by (64)-(66), is the stationary distribution of the process $\{(E(t), X(t))\}$.

The potentials $m\left(y, x \mid e_{i}\right)$ for the above theorem are computed using Lemma 3 and Proposition 2 , and the $(K+1)(N+1) \times(K+1)(N+1)$ system of balance equations is reduced to the $(N+1) \times(N+1)$ system $(64)$.

\section{Conclusion}

Our focus in this work was to study the stationary behaviour of a continuous-time Markov chain in a random environment. We have proved necessary and sufficient conditions for a product-form stationary distribution, in Section 3. However, as was clear from the statements of the theorems and their applications in queueing models, presented in Section 5, the class of 
models that have product-form stationary distributions is very limited. On the other hand, the inversion formula, which gives the stationary distribution when the Palm distributions at certain environmental transitions are known, has universal applicability in the class of continuous-time Markov chains in random environments. The inversion formula and its consequences seem to be efficient tools for the study of a model in this class. First, they are useful in simulation studies, since they make it possible to observe the system only during environmental changes and deduce conclusions for its equilibrium behaviour in continuous time. More importantly, they provide an (equivalent) alternative to the system of balance equations (24), which is more readily solved in many cases. We have given some examples of this approach in Section 6, but the overall method requires further investigation. We think that the first step in this direction is to combine this approach with the matrix-analytic or spectral decomposition methods in the case where the continuous-time Markov chain in a random environment is a quasi-birthdeath process (and perhaps nonhomogeneous). It also seems important to deduce bounds and approximations for some stationary characteristics, using information about certain Palm distributions. An asymptotic analysis using this approach, for systems with very high or very low environmental change rates, also seems possible.

\section{Acknowledgements}

The author was supported by the University of Athens grant ELKE/70/4/6415 and by the Greek Ministry of Education and European Union Program Pythagoras.

\section{References}

Anisimov, V. AND SzTrik, J. (1989). Asymptotic analysis of some complex renewable systems operating in random environments. Europ. J. Operat. Res. 41, 162-168.

Bourgin, R. D. AND Cogburn, R. (1981). On determining absorption probabilities for Markov chains in random environments. Adv. Appl. Prob. 13, 369-387.

Chang, C.-S. And Nelson, R. (1993). Perturbation analysis of the M/M/1 queue in a Markovian environment via the matrix-geometric method. Commun. Statist. Stoch. Models 9, 233-246.

Chao, X., Pinedo, M. and Miyazawa, M. (1999). Queueing Networks: Negative Customers, Signals and Product Form. John Wiley, New York.

ÇInlar, E. (1975). Introduction to Stochastic Processes. Prentice-Hall, Englewood Cliffs, NJ.

Cogburn, R. (1991). On the central limit theorem for Markov chains in random environments. Ann. Prob. 19, $587-604$.

Cogburn, R. AND Torrez, W. C. (1981). Birth and death processes with random environments in continuous time. J. Appl. Prob. 18, 19-30.

El-Taha, M. And Stidham, S., JR. (1999). Sample-Path Analysis of Queueing Systems. Kluwer, Boston, MA.

FAKINOS, D. (1982). The generalized M/G/k blocking system with heterogeneous servers. J. Operat. Res. Soc. 33, 801-809.

FAKINOS, D. (1991). Insensitivity of generalized semi-Markov processes evolving in a random environment. J. Operat. Res. Soc. 42, 1111-1115.

FAKINOS, D. AND ECONOMOU, A. (1998). Overall station balance and decomposability for non-Markovian queueing networks. Adv. Appl. Prob. 30, 870-887.

FALIN, G. (1996). A heterogeneous blocking system in a random environment. J. Appl. Prob. 33, 211-216.

Gaver, D. P., Jacobs, P. A. AND Latouche, G. (1984). Finite birth-and-death models in randomly changing environments. Adv. Appl. Prob. 16, 715-731.

Gupta, P. L. AND Gupta, R. D. (1990). A bivariate random environmental stress model. Adv. Appl. Prob. 22, $501-503$.

Hambly, B. (1992). On the limiting distribution of a supercritical branching process in a random environment. J. Appl. Prob. 29, 499-518.

HeLM, W. E. AND WALDMANN, K.-H. (1984). Optimal control of arrivals to multiserver queues in a random environment. J. Appl. Prob. 21, 602-615.

Karlin, S. And McGregor, J. L. (1965). Ehrenfest urn models. J. Appl. Prob. 2, 352-376.

Kelly, F. P. (1979). Reversibility and Stochastic Networks. John Wiley, New York.

Lefèvre, C. AND Milhaud, X. (1990). On the association of the lifelengths of components subjected to a stochastic environment. Adv. Appl. Prob. 22, 961-964. 
Melamed, B. and Yao, D. D. (1995). The ASTA property. In Advances in Queueing (Prob. Stoch. Ser.), ed. J. H. Dshalalow, CRC, Boca Raton, FL, pp. 195-224.

Mitrani, I. AND ChaKKa, R. (1995). Spectral expansion solution for a class of Markov models: application and comparison with the matrix-geometric method. Perf. Eval. 23, 241-260.

Neuts, M. F. (1981). Matrix-Geometric Solutions in Stochastic Models. An Algorithmic Approach (Johns Hopkins Ser. Math. Sci. 2). Johns Hopkins University Press, Baltimore, MD.

Norris, J. R. (1997). Markov Chains. Cambridge University Press.

NúÑEZ-QueIJA, R. (1997). Steady-state analysis of a queue with varying service rate. Res. Rep. PNA-R9712, CWI.

O'Cinneide, C. A. And Purdue, P. (1986). The M/M/ $\infty$ queue in a random environment. J. Appl. Prob. 23, $175-184$.

Posner, M. J. M. and Zuckerman, D. (1990). Optimal R \& D programs in a random environment. J. Appl. Prob. 27, 343-350.

Serfozo, R. (1999). Introduction to Stochastic Networks. Springer, New York.

Stidham, S., JR. And El-Taha, M. (1995). Sample-path techniques in queueing theory. In Advances in Queueing (Prob. Stoch. Ser.), ed. J. H. Dshalalow, CRC, Boca Raton, FL, pp. 119-166.

SzTrIK, J. (1987). On the heterogeneous M/G/n blocking system in a random environment. J. Operat. Res. Soc. 38, 57-63.

Tsitsiashvili, G. Sh., Osipova, M. A., Koliev, N. V. And Baum, D. (2002). A product theorem for Markov chains with application to PF-queueing networks. Ann. Operat. Res. 113, 141-154.

Van Assche, W., Parthasarathy, P. R. and Lenin, R. B. (1999). Spectral representation of four finite birth and death processes. Math. Scientist 24, 105-112.

Van DiJK, N. M. (1993). Queueing Networks and Product Forms: A System Approach. John Wiley, Chichester.

YamaZaKi, G. And MiYaZawa, M. (1995). Decomposability in queues with background states. Queueing Systems 20, 453-469.

ZHU, Y. (1991). A Markov-modulated M/M/1 queue with group arrivals. Queueing Systems 8, 255-263.

ZHU, Y. (1994). Markovian queueing networks in a random environment. Operat. Res. Lett. 15, 11-17. 